\title{
Black Hole Accretion in Low States: Electron Heating
}

\author{
Siming Liu*, Christopher L. Fryer ${ }^{\dagger}$ and Hui $\mathrm{Li}^{\dagger}$ \\ *Key Laboratory of Dark Matter and Space Astronomy, Purple Mountain Observatory, Chinese Academy of Sciences, \\ Nanjing, 210008, P. R. China \\ ${ }^{\dagger}$ Los Alamos National Laboratory, Los Alamos, New Mexico 87545, USA
}

\begin{abstract}
Plasmas in an accretion flow are heated by magneto-hydrodynamic turbulence generated through the magnetorotational instability (MRI). The viscous stress driving the accretion is intimately connected to the microscopic processes of turbulence dissipation. We show that, in a few well-observed black hole accretion systems, there is compelling observational evidence of efficient electron heating by turbulence or collective plasma effects in low accretion states, when Coulomb collisions are not efficient enough to establish a thermal equilibrium between electrons and ions at small radii. We consider a Keplerian two-temperature accretion flow with a constant mass accretion rate in the pseudoNewtonian gravitational potential and take into account the bremsstrahlung, synchrotron, and inverse Comptonization cooling processes. The balance of gravitational energy dissipation and turbulence energy cascade requires that the viscous stress be proportional to the product of the turbulence kinetic energy density and the total turbulence energy density, which may contradict the result of some shearing box simulations that the viscous stress is proportional to the magnetic field energy density. The critical mass accretion rate, below which the two-temperature solution may exist, is determined by the cooling processes and the collisional energy exchanges between electrons and ions and has very weak dependence on the collision-less heating of electrons by turbulence, which becomes more important at lower accretion rates. If the collision-less heating is dominated by the transit-time damping processes, small scale waves propagating obliquely with respect to the large scale magnetic field are prohibited, which may affect the saturate state of the MRI driven turbulence significantly. The plasma also needs to be strongly magnetized with the magnetic field and proton energy densities comparable so that electrons can share more of the dissipated gravitational energy. The heating of relativistic electrons is efficient since the heating rate is proportional to the mean momentum of the particles, and the electron heating may also be enhanced by their resonant scattering with small scale nearly parallel propagating waves.
\end{abstract}

Keywords: Acceleration of particles, accretion, accretion disks, black hole physics, plasmas, radiation mechanisms: thermal, turbulence.

\section{INTRODUCTION}

Black hole accretion is one of the most powerful energy sources in the universe. When the luminosity of the system is close to the Eddington luminosity, the accretion can be described by the classical Shakura-Sunyaev disk [1], which produces a multi-color blackbody radiation with the flux and temperature determined by the black hole mass and accretion rate. Observations of galactic black hole X-ray binaries strongly support such a scenario [2]. The less energetic nonthermal high energy emission component frequently observed has been attributed to hot magnetized coronas above the disk [3, 4].

The magneto-rotational instability (MRI) has been generally accepted as the basic mechanism producing the less well-understood turbulence viscosity that drives the accretion [5-7]. The dissipated gravitational energy is first converted into magneto-hydrodynamic (MHD) turbulence, which then heats the accretion flow through viscous and Ohmic dissipations. In these optically thick slim disks, the Coulomb collision time scales are much shorter than other

*Address correspondence to this author at the Purple Mountain Observatory, Chinese Academy of Sciences, 2 West Beijing Road, Nanjing, 210008, China; Tel: 8625 83332173; Fax: 8625 83332173;

E-mail: liusm@pmo.ac.cn relevant time scales, electrons and ions reach a thermal equilibrium so that the observed emission can be welldescribed with blackbody spectra modified by the radiation transfer through the disk structure. The acceleration and/or heating of electrons by turbulence can at most be constrained with observations of the non-thermal component emitted from the collision-less coronas [8, 9].

Many black hole candidates are often observed in a super- or sub- Eddington emission state, and there are many distinct observational phenomena, such as relativistic outflows and quasi-periodic oscillations, that have not been well understood though many models have been proposed [10-13]. Both theoretical investigations and observations suggest that, below a critical mass accretion rate $\dot{M}_{\mathrm{cr}}$, a twotemperature accretion flow likely develop near the black hole due to the much higher radiation efficiency of electrons than ions and inefficient Coulomb coupling between them so that the local thermal equilibrium cannot be established between the two [6, 14-17]. Such a two-temperature flow is also expected due to the fact that charged particles reach a thermal equilibrium with their kind much faster than with others through Coulomb collisions [18]. This paper studies the electron heating processes in these low states. 
Besides energy exchanges through Coulomb collisions, charged particles can also be energized by plasma waves through collision-less processes. These energization processes will directly affect the characteristics of the observed emission and therefore play crucial roles in our study of these systems in the low states. As pointed out by Bisnovatyi-Kogan and Lovelace [19], the Ohmic heating of electrons through dissipation of MHD turbulence can be very efficient. Quataert [20] studied the electron and proton heating through the transit-time damping processes. Sharma et al. [21] recently found that electrons might also be energized in the dynamo processes of magnetic field amplification through the MRI. However, the complexity of processes in a turbulent plasma has made the collision-less electron heating by collective plasma effects a difficult problem [15]. In these theoretical investigations, certain assumptions have to be made on the coupling between charged particles and the turbulent electromagnetic fields to derive some quantitative results, which are usually sensitive to the prior assumptions.

The collision-less electron heating by turbulent magnetic fields is often ignored in most phenomenological models [14]. In the advection dominated accretion flow models, it is simply assumed that a small fraction of the energy dissipated through viscosity is converted into electrons [22, 23] suggesting that this is a trivial process. Alternatively, onetemperature models have been proposed for Sagittarius A*, the compact radio source associated with the low-luminosity supermassive black hole in the Galactic Center [24, 25], assuming that electrons and protons are coupled by turbulence effectively [26, 27].

On the other hand, the collision-less electron heating processes may be constrained by observations of the relevant systems. Recent studies of flares from Sagittarius A* indicate that electrons can be heated efficiently by MHD turbulence and the distribution of relativistic electrons under the influence of a turbulent magnetic field can be approximated as relativistic Maxwellian [28-31]. The same processes may also play important roles in the heating of electrons in the two-temperature accretion flows. In this paper, we consider a Keplerian accretion disk model and the dominant cooling processes in a fully ionized magnetized plasma. We show that Coulomb collisions with ions cannot heat electrons efficiently and an extra electron heating process is required to explain observations of Sagittarius A* and the galactic X-ray binary Cygnus X-1 [32]. This is in contradiction with what is suggested in some of the previous studies. Magnetic turbulence could play such a role, and we believe that the collision-less heating of electrons by MHD turbulence should not be ignored in any quantitative theoretical modeling of these low accretion states.

The basic equations for the turbulent accretion disk are given in $\S 2$. In $\S 3$, we discuss the energy flow in this system and show how the energy conservation can be used to connect the turbulence viscosity, turbulence cascade, and microscopic energy dissipation processes and study their implications. The electron heating processes are studied in $\S$ 4 and it is shown that observations of Sagittarius $A^{*}$ in the millimeter and sub-millimeter range are difficult to explain without introducing efficient electron heating by turbulence. The cooling processes are studied in $\S 5$ and the model is applied to galactic X-ray binaries in $\S 6$. In $\S 7$, we draw conclusions and discuss the model limitations and possible improvements in the future.

\section{BASIC EQUATIONS FOR KEPLERIAN TURBU- LENT ACCRETION FLOWS IN THE PSEUDO- NEWTONIAN POTENTIAL}

We consider a fully ionized hydrogen plasma. Then the gas pressure and the thermal energy density are given, respectively, by

$$
\begin{aligned}
& P=n k_{\mathrm{B}}\left(T_{p}+T_{e}\right), \\
& \mathscr{E}=n k_{\mathrm{B}}\left(1.5 T_{p}+\alpha T_{e}\right),
\end{aligned}
$$

where the gas density, the proton and electron temperatures are denoted by $n, T_{p}$ and $T_{e}$, respectively, $k_{\mathrm{B}}$ is the Boltzmann constant, and $\alpha=x\left[3 K_{3}(x)+K_{1}(x)-4 K_{2}(x)\right] / 4 K_{2}(x)$ with $x=m_{e} c^{2} / k_{\mathrm{B}} T_{e}$, where $m_{e}$ and $c$ denote the electron mass and the speed of light, respectively, and $K_{i}$ refers to the $i$ th order modified Bessel function. $K_{i}(x) \rightarrow 2^{i-1}(i-1) ! / x^{i}$ as $x \rightarrow 0$ and $K_{i}(x) \rightarrow(\pi / 2 x)^{1 / 2} \exp (-x)\left[1+\left(4 i^{2}-1\right) / 8 x\right]$ as $x \rightarrow \infty$. In the pseudo-Newtonian gravitational potential [33], the potential and Keplerian angular velocity are given, respectively, by

$$
\begin{aligned}
& \varphi=-\frac{G M}{r-r_{\mathrm{s}}}, \\
& \Omega_{\mathrm{K}}=\left[\frac{G M}{r\left(r-r_{\mathrm{S}}\right)^{2}}\right]^{1 / 2},
\end{aligned}
$$

where the gravitational constant, the black hole mass and the radius are denoted by $G, M$ and $r$ respectively, and $r_{\mathrm{S}}=2 G M / c^{2}$ is the Schwarzschild radius of the black hole.

Due to the decoupling between electrons and ions, the radiation efficiencies of two-temperature accretion flows will not be as high as the optically thick slim disks. The accretion processes likely drive strong winds from the hot disk [34], and there are currently no strong observational constraints on these processes. To simplify our model we consider the steady state properties of the accretion flow averaged in the vertical direction and assume a radius independent accretion rate, i.e., ignoring the effects of winds:

$\dot{M}=-4 \pi r H v_{r} n\left(m_{p}+m_{e}\right)$,

where $v_{r}$ and $H$ are the radial velocity and the scale height of the accretion flow, respectively, and $m_{p}$ is the proton mass. The vertical structure of the disk can be very complicated as suggested by MHD simulations [35] and may be essential to explain the observed non-thermal high energy emission in low states $[8,9]$. It, however, is not expected to introduce significant changes to our quantitative results 
below, which deal with the energetically dominant thermal emission component from the main body of the hot accretion flow [14].

MHD simulations show that the azimuthal velocity of the flow is given by the Keplerian velocity $v_{\mathrm{K}}=r \Omega_{\mathrm{K}}$ [35], which requires that the inner boundary radius, $r_{i}$, should be greater than $3 r_{\mathrm{S}}$, the radius of the last stable orbit. We also assume that the angular momentum flux through the disk is a small fraction $(\delta$ ) of that carried by the Keplerian accretion flow inward at the last stable orbit [34], as expected for strongly magnetized disks [36]. From the angular momentum conservation of the accretion flow, we then have

$$
\begin{aligned}
& v_{r}=v \frac{\mathrm{d} \ln \Omega_{\mathrm{K}}}{\mathrm{d} r}\left[1-\delta \frac{r_{i}^{2} \Omega_{\mathrm{K}}\left(r_{i}\right)}{r^{2} \Omega_{\mathrm{K}}}\right]^{-1}=-v\left(\frac{1}{2 r}+\frac{1}{r-r_{\mathrm{S}}}\right) \\
& {\left[1-\delta \frac{r_{i}^{3 / 2}\left(r-r_{\mathrm{S}}\right)}{r^{3 / 2}\left(r_{i}-r_{\mathrm{S}}\right)}\right]^{-1}}
\end{aligned}
$$

where the kinematic viscosity

$$
v=\frac{2 \beta_{p} \beta_{v} k_{\mathrm{B}}\left(T_{p}+T_{e}\right)\left(r-r_{\mathrm{S}}\right)^{2} r^{1 / 2}}{\left(m_{p}+m_{e}\right)\left(3 r-r_{\mathrm{S}}\right)(G M)^{1 / 2}},
$$

and in accord with Melia et al. [27] $\beta_{p}=\left\langle B^{2}\right\rangle / 8 \pi\langle P\rangle$, where, and in what follows, " \langle\rangle $"$ indicates the ensemble average of the corresponding quantities, and $\beta_{v}$ is defined as the ratio of the total average stress to the average magnetic field energy density $\left\langle B^{2} / 8 \pi\right\rangle$, which we assume is much higher than the energy density of any preexisting large scale magnetic fields. ${ }^{1}$ Then the radial velocity can be rewritten as

$v_{r}=\frac{2 \beta_{v} \beta_{p} k_{\mathrm{B}}\left(T_{p}+T_{e}\right)\left(r-r_{\mathrm{S}}\right)}{f\left(m_{p}+m_{e}\right)(G M r)^{1 / 2}}$.

where $f \equiv\left[1-\delta r_{i}^{3 / 2}\left(r-r_{\mathrm{S}}\right) / r^{3 / 2}\left(r_{i}-r_{\mathrm{S}}\right)\right]$. As expected, this radial velocity diverges at $r_{i}$ for $\delta=1$ as required by the zero stress inner boundary condition. In general, $\delta<1$, there is no singularity in the radial velocity profile.

Since the magnetic field is dominated by the toroidal component, one has

$$
\begin{aligned}
& H=\left[\frac{r k_{\mathrm{B}}\left(T_{p}+T_{e}\right)\left(1+\beta_{p}+\beta_{t}\right)}{G M\left(m_{p}+m_{e}\right)}\right]^{1 / 2}\left(r-r_{\mathrm{S}}\right), \\
& n=\frac{f G M M\left(m_{p}+m_{e}\right)^{1 / 2}}{4 \pi \beta_{v} \beta_{p}\left[k_{\mathrm{B}}\left(T_{p}+T_{e}\right)\right]^{3 / 2}\left(1+\beta_{p}+\beta_{t}\right)^{1 / 2} r\left(r-r_{\mathrm{S}}\right)^{2}},
\end{aligned}
$$

where $\beta_{t}=\left\langle n\left(m_{e}+m_{p}\right) u^{2}\right\rangle /\langle P\rangle \quad$ is the ratio of the turbulence ram pressure $\left\langle n\left(m_{e}+m_{p}\right) u^{2}\right\rangle$ to the gas pressure, and $3^{1 / 2} u$ is the eddy speed at the coherent length scale

${ }^{1}$ Note that the viscous parameter in the classical Shakura-Sunyaev disk model $\alpha=\beta_{v} \beta_{p}$. $\sim H$ of the turbulence flow. Because the compressible component of MHD turbulence carries much less energy than the solenoidal part, which corresponds to the eddy turnovers, we do not treat these two components separately and use $u$ to characterize the turbulence effects [37]. The turbulence motion produces pressure and carries energy similar to an ideal gas with $u$ an analogy to the isothermal sound speed $c_{S}=\left[P / n\left(m_{e}+m_{p}\right)\right]^{1 / 2}$. Thus the kinetic energy carried by the turbulence is given by $1.5 n\left(m_{e}+m_{p}\right) u^{2}$. For the Keplerian turbulent accretion flow with the viscosity induced by the MRI, there is an intimate connection between $\beta_{p}$ and $\beta_{t}$. Local shearing box MHD simulations of accretion flows indicate that $\beta_{p} \simeq 3$ - $4 \beta_{t}$ [38]. Pessah et al. [39] show that, when the large scale magnetic field is weak, the Reynolds stress is about 5 times smaller than the Maxwell stress, i.e., $-3\left\langle u_{r} u_{\varphi}\right\rangle_{\simeq}-\left\langle B_{r} B_{\varphi}\right\rangle / 20 \pi n\left(m_{e}+m_{p}\right) \simeq \beta_{v} \beta_{p} c_{S}^{2} / 6 \leq\left\langle 3 u^{2} / 2\right\rangle$, where the subscripts $r$ and $\varphi$ indicate the components of the corresponding vectors. Then one has $\beta_{v} \beta_{p} \leq 9 \beta_{t}$. They also find that $\beta_{v} \simeq 0.7$, which implies that $\beta_{p} \leq 12.9 \beta_{t}$ in agreement with simulations of Hawley et al. [38]. However, for turbulent magnetic fields generated through turbulence driven at large scales, depending on the strength of the large scale magnetic field the kinetic energy density can be one order of magnitude higher the magnetic field energy density implying $\beta_{p} \sim 0.1-1.0 \beta_{t}[40,41]$.

The energy conservation equation is given by

$\frac{\mathrm{d}}{H r \mathrm{~d} r}\left\{H_{r} v_{r}\left[P\left(1+2 \beta_{p}+2.5 \beta_{t}\right)+\mathscr{E}+n\left(m_{p}+m_{e}\right)\right.\right.$

$\left.\left.{ }^{1}\left[\phi+0.5(1-2 f) v_{\mathrm{K}}^{2}+0.5 v_{r}^{2}\right]\right]\right\}=-\Lambda$,

where $\Lambda$ is the radiative cooling rate, and we have ignored any energy fluxes carried away from the disk by winds and waves and taken into account the effects of magnetic fields properly [27]. The power density generated by the viscous torque force is given by $-\mathrm{d}\left[f H r v_{r} n\left(m_{p}+m_{e}\right) v_{\mathrm{K}}^{2}\right] / H r \mathrm{~d} r$. This equation can be put as

$\frac{\mathrm{d}}{\mathrm{d} r} \varepsilon=-\frac{\Lambda}{v_{r} n}$,

where

$$
\begin{aligned}
& \varepsilon=k_{\mathrm{B}}\left[T_{e}\left(\alpha+1+2 \beta_{p}+2.5 \beta_{t}\right)+T_{p}\left(2.5+2 \beta_{p}+2.5 \beta_{t}\right)\right]+ \\
& \left(m_{p}+m_{e}\right)\left[\varphi+0.5(1-2 f) v_{\mathrm{K}}^{2}+0.5 v_{r}^{2}\right]
\end{aligned}
$$

can be considered as the energy of the accretion flow per proton and $\dot{M} \varepsilon / m_{p}$ gives the inwardly directed energy flux through the accretion disk [34]. Because our results beyond $\sim 10 r_{S}$ have very weak dependence on the parameter $\delta$ or $f$, which mostly affects the disk structure near the black hole, where the effects of outflows and black hole spins are important, in what follows we will ignore the angular 
momentum flux through the disk, i.e., $\delta=0$ and $f=1$. We focus on studying the collision-less electron heating by MHD turbulence and leave quantitative modeling of the disk structure and outflows at small radii to a separate investigation, which will adopt the modified pseudoNewtonian potential with the black hole spin effects incorporated [42].

\section{ANOMALOUS VISCOSITY, MHD TURBULENCE, AND DAMPING OF PLASMA WAVES}

Because the viscous stress driving the accretion is induced by the MRI [7], the gravitational energy dissipation first generates MHD turbulence, which then heats the gas as the turbulence cascades from large scales to small scales. A fraction of the turbulence energy may also be carried away from the disk by MHD waves propagating toward large radii. However, this process is not expected to dominate the dynamics of the accretion flow at least for the relative more powerful states, when the hard X-ray luminosity of X-ray binaries can be a significant fraction of the Eddington luminosity. Very high accretion rates will be needed to produce the observed X-ray power if waves carry most of the dissipated gravitational energy toward large radii. The turbulence cascade also needs to be suppressed dramatically to make the wave escape process dominant.

In the steady state, the viscous heating rate

$\Gamma_{v}=v_{r} n\left(m_{p}+m_{e}\right) \frac{\mathrm{d}\left[-\varphi+0.5 v_{\mathrm{K}}^{2}\right]}{\mathrm{d} r}=-v_{r} n\left(m_{p}+m_{e}\right) \frac{G M\left(3 r-r_{S}\right)}{2\left(r-r_{S}\right)^{3}}$

which includes contributions from both the viscous and gravitational forces, should be slightly greater than the turbulence energy cascade rate [43-46].

$\Gamma_{c}=C_{1} n\left(m_{p}+m_{e}\right) \frac{\left(1.5 u^{2}+0.5 v_{A}^{2}\right) u^{2}}{H\left(v_{\mathrm{A}}+u+H^{2} \Omega_{\mathrm{K}} / r\right)}$

where $C_{1} \sim 1$ is dimensionless and depends on the anisotropy of the turbulence, and $v_{\mathrm{A}}=\left[\left\langle B^{2}\right\rangle / 4 \pi\langle n\rangle\left(m_{e}+m_{p}\right)\right]^{1 / 2}$ is the Alfvén speed. We will impose the constraint $\Gamma_{v} \geq \Gamma_{c} \geq 0.5 \Gamma_{v}$ in what follows. Due to the shearing motion of the large scale flow, the turbulence is highly anisotropic and the turbulence energy may be carried mostly by the turbulent magnetic fields [38]. Plasma waves in a turbulent flow can contribute energy to both the kinetic motion and the magnetic field and are not independent energy components. Given the high energy dissipation rate of shock waves, the turbulence should be subsonic in the quasi-equilibrium state of the accretion flows, i.e.,

$$
\begin{aligned}
& u \leq c_{S}=\left[\frac{k_{\mathrm{B}}\left(T_{p}+T_{e}\right)}{m_{p}+m_{e}}\right]^{1 / 2}=\left(\frac{1}{1+\beta_{p}+\beta_{t}}\right)^{1 / 2} H \Omega_{\mathrm{K}}= \\
& \left(2 \beta_{p}\right)^{-1 / 2} v_{\mathrm{A}}=\beta_{t}^{-1 / 2} u .
\end{aligned}
$$

One then has $\beta_{t} \leq 1$. Because the longitudinal sound waves may not be excited in subsonic turbulence, they don't contribute to the anomalous viscosity. We assume that such waves do not play important roles in the accretion flow and therefore do not affect the cascade of MHD turbulence. Here we only consider the propagation effects of transverse waves with a characteristic phase velocity of $\sim v_{\mathrm{A}}$ and the shearing effects on the cascade of MHD turbulence.

To demonstrate the implications of turbulence cascade on the dynamics of accretion flows, we first consider a nonradiative accretion flow with $\Lambda=0$. At large radii $\left(r>>r_{S}\right)$, we can ignore the kinetic energy associated with the radial motion and $\alpha=1.5$. Then we have the following solution:

$$
\begin{aligned}
& c_{S}=\left[3 /\left(5+5 \beta_{t}+4 \beta_{p}\right)\right]^{1 / 2} v_{\mathrm{K}}, \\
& H=\left[3\left(1+\beta_{p}+\beta_{t}\right) /\left(5+5 \beta_{t}+4 \beta_{p}\right)\right]^{1 / 2} r, \\
& v_{r}=-\left[3 \beta_{v} \beta_{p} /\left(5+5 \beta_{t}+4 \beta_{p}\right)\right] v_{\mathrm{K}}, \\
& n=\left[3 /\left(5+5 \beta_{t}+4 \beta_{p}\right)\right]^{-3 / 2}\left(1+\beta_{p}+\beta_{t}\right)^{-1 / 2} \\
& \dot{M} / 4 \pi \beta_{v} \beta_{p}\left(m_{p}+m_{e}\right) r^{2} v_{\mathrm{K}} .
\end{aligned}
$$

For the two-temperature accretion flow we are interested in, the disk structure will be similar to these due to the low radiation efficiency. These analytical results are also very helpful to understand the quantitative investigations in the following sections.

From $2 \Gamma_{c} \geq \Gamma_{v} \geq \Gamma_{c}$, one gets the viscous parameter

$$
\beta_{v} \beta_{p} \geq \frac{C_{1} \beta_{t}\left(\beta_{t}+2 \beta_{p} / 3\right)}{\left(1+\beta_{p}+\beta_{t}\right)^{1 / 2}\left\{\left(2 \beta_{p}\right)^{1 / 2}+\beta_{t}^{1 / 2}+\left(1+\beta_{p}+\beta_{t}\right)\left[3 /\left(5+5 \beta_{t}+4 \beta_{p}\right)\right]^{1 / 2}\right\}} \geq 0.5 \beta_{v} \beta_{p} .
$$

For $\beta_{v}=0.7$ and $\beta_{p}=3.5 \beta_{t}$, one has

$0.735\left(\beta_{v} / 0.7\right) \geq \frac{C_{1}}{\left(\beta_{t}^{-1}+4.5\right)^{1 / 2}\left[3.65 \beta_{t}^{-1 / 2}+0.775\left(\beta_{t}^{-1}+4.5\right)\left(\beta_{t}^{-1}+3.8\right)^{-1 / 2}\right]} \geq 0.368\left(\beta_{v} / 0.7\right)$.

When $\beta_{t}<<1$, one has $3.25\left(\beta_{v} / 0.7\right) \geq C_{1} \beta_{t} \geq 1.63\left(\beta_{v} / 0.7\right)$. For strong MHD turbulence with $\beta_{t}=\beta_{p}=1$, we have $0.5 C_{1} \geq \beta_{v} \geq 0.25 C_{1}$. For $\beta_{v}=0.7$, we have $2.8 \geq C_{1} \geq 1.4$. In general, for radiatively inefficint accretion flows with $\beta_{t} \leq \beta_{p} \leq 1$ and $C_{1} \sim 1$, the suppression of the turbulence cascade is dominated by the shearing effect, and $\beta_{v}$ should be proportional to $\beta_{t}$. Therefore the viscous stress should be proportional to the product of the turbulence energy density and the ratio of the turbulence kinetic energy density to the gas pressure. These results are simple consequences of the balance between the gravitational energy release and the transfer of this energy into the gas through the turbulence cascade, which is valid as far as energy dissipation through the turbulence cascade dominates. 
Shearing box MHD simulations of non-radiative accretion flows by Pessah et al. [39] suggest that $\beta_{v}=0.7$ is independent of $\beta_{p}$, implying a $\beta_{t}$ independent of $\beta_{p}$. This is a bit surprising given the amplitudes of velocity and magnetic field fluctuations of the most unstable MRI mode are correlated [7, 27]. It is possible that this correlation does not exist any more in the saturated turbulence state. In this case, we expect a constant level of the turbulence kinetic motions and that the ratio of the turbulence kinetic and magnetic energy densities decreases with the increase of $\beta_{p}$. If the ratio of the Reynolds stress to the Maxwell stress is also independent of $\beta_{p}$ [39], we expect that the ratio of Reynolds stress to the turbulence kinetic energy density increases with the increase of the turbulence magnetic energy density. With the increase of $\beta_{p}$, the structure of the turbulence velocity field, as indicated by the ratio of the Reynolds stress to the turbulence kinetic energy density, will change significantly while the radio of Maxwell stress to the magnetic field energy density remains constant. However, the Reynolds stress is less than the turbulence kinetic energy density, implying an upper limit for $\beta_{p}$ or a change of the ratios of the Maxwell stress to the Reynolds stress and/or the magnetic field energy density. With the decrease of $\beta_{p}, \beta_{t}$ may exceed $\beta_{p}$ so that the turbulence is dominated by the kinetic motions, which hasn't been observed in shearing box MHD simulations. This is also in conflict with the dominance of the viscous stress by the Maxwell stress, which is less than the magnetic field energy density. Since the viscous stress needs to be proportional to the product of the turbulence kinetic energy density and the total turbulence energy density, in the kinetic energy dominant regime, the stress cannot be dominated by the Maxwell stress. These predictions can be verified with further MHD simulations.

For a hydrodynamic turbulence with $\beta_{p} \ll 1$, $\beta_{v} \beta_{p}=-\left\langle u_{r} u_{\varphi}\right\rangle / c_{S}^{2}<u^{2} / 2 c_{S}^{2}=\beta_{t} / 2$. One has

$C_{1}<0.5\left(1+\beta_{t}^{-1}\right)^{1 / 2}+0.5(3 / 5)^{1 / 2}\left(1+\beta_{t}^{-1}\right)$.

For $\beta_{t}=1$, one has $C_{1}<1.48$. Although this constraint on $C_{1}$ is derived for a non-radiative flow with strong hydrodynamic turbulence, it should be true in general except that the scaling relation for the turbulence energy cascade (15) has been modified significantly due to the anisotropy of the large scale turbulence. On the other hand, if the viscosity is dominated by the Maxwell stress with $\beta_{p} \gg \beta_{t}$, then $\beta_{v} \beta_{p}=-\left\langle B_{r} B_{\varphi}\right\rangle / 4 \pi n\left(m_{e}+m_{p}\right) c_{S}^{2}<B^{2} / 8 \pi\langle P\rangle=\beta_{p}$. One has $\beta_{v}<1$, and $C_{1}<1.5 \beta_{t}^{-1}\left(1+\beta_{p}\right)^{1 / 2}\left\{\left(2 \beta_{p}\right)^{1 / 2}+\left(1+\beta_{p}\right)\left[3 /\left(5+4 \beta_{p}\right)\right]^{1 / 2}\right\}$.

This constraint is not as strict as that for the hydrodynamic case because the cascade rate is proportional to the eddy speed squared. We notice that the kinetic viscosity should scale as $u^{2}$ instead of $u$ as proposed originally by Shakura and Sunyaev [1] because the dynamical time in the shearing flow $\sim \Omega_{\mathrm{K}}^{-1}=H / c_{F}$ is shorter than the eddy turnover time $\sim \boldsymbol{H} / \boldsymbol{u}$, which reduces the effective turbulent viscosity to $u^{2} H /\left(c_{F}+u\right)$. The Shakura and Sunyaev's formula is recovered when $u \geq c_{F}$, i.e., for the highly dissipative supersonic turbulence.

For the two-temperature collision-less plasma we are interested in, magnetic fields play important roles in producing the turbulence and anomalous viscosity, and we expect $v_{\mathrm{A}} \geq u$ implying $2 \beta_{p}>\beta_{t}$. Due to the shearing motion of the Keplerian accretion flow, the turbulent magnetic field is dominated by the azimuthal component $[27,35]$. To better understand this anisotropic MHD turbulence, one needs to model the collision-less damping of the plasma waves by the background particles. As shown by Petrosian et al. [47], the transit-time damping (TTD) dominates the collision-less thermal damping of transverse waves with the damping rate for isotropic particle distributions:

$$
\begin{aligned}
& \Gamma_{k}=\frac{\pi^{1 / 2} k_{\sin ^{2} \theta}}{2\left(m_{e}+m_{p}\right) \cos \theta}\left[\left(2 k_{\mathrm{B}} T_{e} m_{e}\right)^{1 / 2} \exp \left(-\frac{m_{e} \omega^{2}}{2 k_{\mathrm{B}} T_{e} k_{\|}^{2}}\right)+\right. \\
& \left.\left(2 k_{\mathrm{B}} T_{p} m_{p}\right)^{1 / 2} \exp \left(-\frac{m_{p} \omega^{2}}{2 k_{\mathrm{B}} T_{p} k_{\|}^{2}}\right)\right],
\end{aligned}
$$

where $\omega, k, \theta$, and $k_{\|}=k \cos \theta$ are the wave frequency, wave number, the angle between the wave vector and the mean large scale magnetic field, and the parallel (to the mean large scale magnetic field) component of the wave vector $k$, respectively. The first and second terms on the right hand side correspond to the TTD by electrons and protons, respectively. Therefore the relative heating rate of electrons and protons through the TTD only depends on the particle temperatures and the phase velocities of the heating waves.

In the two temperature accretion flow, we expect that $c_{S}>v_{A}>u$. If $T_{e}>>T_{p}$, ion acoustic waves can be excited.

The consequent heating of protons by these waves will make the electron and proton temperatures comparable. The TTD is therefore dominated by protons [20]. For $\beta_{p} \leq 1$, the TTD rate is comparable to the the growth rate of the unstable MRI modes [7, 27] except for the nearly parallel or perpendicular propagating waves. The latter is subject to damping by magnetic field wandering. Therefore only large scale nearly parallel propagating waves may be excited. After the instabilities develop the flow into a turbulent saturation state, the turbulence cascade follows the Kraichnan phenomenology with the turbulence cascade rate given by $\tau_{\text {cas }}^{-1} \simeq C_{1} u(k)^{2} k /\left[v_{A}+u(k)+\left(1+\beta_{p}+\beta_{t}\right) c_{S}^{2} / v_{K}\right], \quad$ where $3^{1 / 2} u(k)$ is the eddy speed at the scale of $2 \pi / k$. In the inertial range, $u(k)=u(2 \pi / k H)^{1 / 4}[44,46]$. The turbulent power spectrum cuts off sharply when the damping rate becomes comparable to the cascade rate. For $T_{e} \simeq T_{p}$, since 
waves with $k_{\|}<<k$ are subject to damping by magnetic field wandering, we have

$$
\frac{C_{1} u^{2}(k)}{v_{A}+u(k)+\left(1+\beta_{p}+\beta_{t}\right) c_{S}^{2} / v_{\mathrm{K}}} \simeq \frac{\pi^{1 / 2} \sin ^{2} \theta_{c} c_{S}}{2 \cos \theta_{c}},
$$

where $\theta_{c}$ is the critical angle, below which the TTD effect is negligible. For $\beta_{p}<<1$, i.e., $\quad c_{S} \gg v_{A}>u$, $\theta_{c}^{2} \simeq 2 C_{1} u^{2} / \pi^{1 / 2}\left[v_{A}+u+\left(1+\beta_{p}+\beta_{t}\right) c_{S}^{2} / v_{k}\right] c_{S}<<1$ even on the spatial scale $H$. Only waves propagating along the mean large scale magnetic field can exist. For $c_{S} \sim v_{A} \sim u$, the TTD can still cause significant damping of the plasma waves on the scale of $H$ especially for $C_{1} \ll<1$. The TTD becomes more prominent at smaller scales.

The TTD prohibits small scale turbulent fluctuations and may enhance the large scale turbulence structure. To our knowledge, none existing MHD simulations have taken into account this damping effect. It is not clear how the damping will affect the characteristics of the MRI. King et al. [48]. K07 pointed out recently that the viscosity given by MHD simulations of accretion disks is about 1 order of magnitude less than that required to explain observations of accretion flows. They discussed several mechanisms that may reduce the level of viscosity in MHD simulations. The collision-less damping of small scale fluctuations may provide another means to increase the viscosity in simulations since the unstable modes of the MRI may grow to a higher intensity before being saturated by the resulting turbulent motions. Therefore we will not limit our model parameter space to values obtained from simulations but rather use observations to derive reasonable values.

There is therefore an intimate connection between the viscous stress and turbulence dissipation. The above formulas for the TTD assume isotropic particle distributions, which is not well justified for the two temperature flows. Other plasma physics processes may also contribute to the energy dissipation. Theoretically it is not well understood what determines the energy partition between electrons and protons, especially for hot magnetized plasmas in the twotemperature accretion flow of black holes. In the following, we will show how observations of low states of black hole accretion systems may be used to constrain the collision-less electron heating rate by turbulence.

\section{ELECTRON HEATING}

The disk structure can be obtained once one specifies the electron heating and cooling rates and the electron and proton temperatures at the outer boundary. Following Blandford and Eichler [49], we have the electron heating time by the MHD turbulence $[50]^{2}$

\footnotetext{
${ }^{2}$ Since fast mode waves may not be excited in the subsonic flow, charged particles can only scatter with Alfvén and slow mode waves that have a characteristic phase velocity of $: v_{A}$.
}

$$
\tau_{\mathrm{ac}}=\frac{3 C_{2} l_{e}\left\langle v_{e}\right\rangle}{v_{A}^{2}},
$$

where the scattering mean free path of the particles $l_{e} \simeq H$, $C_{2} \sim 1$ is a dimensionless constant, and

$$
\left\langle v_{e}\right\rangle=\frac{2 c(x+1)}{x^{2} K_{2}(x) \exp (x)}
$$

is the mean electron speed. Note that $\left\langle v_{e}\right\rangle / c \rightarrow\left(8 k_{\mathrm{B}} T_{e} / \pi m_{e} c^{2}\right)^{1 / 2}$ as $x=m_{e} c^{2} / k_{\mathrm{B}} T_{e} \rightarrow \infty$ and the electron heating becomes relatively more efficient at smaller radii where electrons may become relativistic and $\left\langle v_{e}\right\rangle$ approaches to $c$. Although cooling processes can also reduce $\left\langle v_{e}\right\rangle$, they actually reduce the heating rate since the heating rate is proportional to the electron temperature, which is proportional to $\left\langle v_{e}\right\rangle^{2}$ for non-relativistic electrons and independent of $\left\langle v_{e}\right\rangle$ in the relativistic case. Then we have

$$
\frac{d T_{e}}{d r}=\frac{T e}{\tau_{\mathrm{ac}} V_{r}}+\frac{T_{p}-T_{e}}{\tau_{\mathrm{Coul}} V_{r}}-\frac{\Lambda}{n \alpha k_{B} V_{r}},
$$

where

$$
\tau_{\text {Coul }}=\frac{3 \pi m_{e} m_{p}\left\langle v_{e}\right\rangle^{3}}{256 n e^{4} \operatorname{In} \lambda},
$$

is the electron-proton energy exchange time through Coulomb collisions, $e$ is the elemental charge unit, and $\ln \lambda \simeq 15$ for most astrophysical situations [18].

There are several ways to estimate $C_{2}$. For the nonradiative accretion flow given by equations (17)-(20) with $\Lambda=0$, if we ignore the Coulomb coupling term, then:

$k_{\mathrm{B}} T_{e}=\left[\pi\left(5+5 \beta_{t}+4 \beta_{p}\right) / 54 C_{2}^{2} \beta_{v}^{2}\left(1+\beta_{p}+\beta_{t}\right)\right]$

$\left(H / l_{e}\right)^{2} m_{e} v_{\mathrm{k}}^{2}$,

$T_{p} / T_{e}=\left[3 /\left(5+5 \beta_{t}+4 \beta_{p}\right)\right]^{2}\left[18 \pi^{-1} C_{2}^{2} \beta_{v}^{2}\left(1+\beta_{p}+\beta_{t}\right)\right]$

$\left(H / l_{e}\right)^{-2}\left(m_{p}+m_{e}\right) / m_{e}-1$

$\stackrel{\sim}{ } 3.79 \times 10^{3}\left(1+\beta_{p}+\beta_{t}\right)\left[C_{2} \beta_{v} l_{e} /\left(1+\beta_{t}+0.8 \beta_{p}\right) H\right]^{2}-1$.

Thus

$C_{2}=1.62 \times 10^{-2}\left[\left(T_{p} / T_{e}+1\right) /\left(1+\beta_{p}+\beta_{t}\right)\right]^{1 / 2}\left(1+\beta_{t}+0.8 \beta_{p}\right) H / \beta_{v} l_{e}$.

It is interesting to note that the proton temperature satisfies an equation similar to equation $(27):^{3}$

$\frac{\mathrm{d} T_{p}}{\mathrm{~d} r}=\frac{T_{p}}{\tau_{\mathrm{ac}} v_{r}}$,

suggesting that the heating time of protons has the same functional form as electrons. Since the mean speed of protons $\left\langle v_{p}\right\rangle$ is usually different from $\left\langle v_{e}\right\rangle$, it is likely that

${ }^{3}$ With the assumptions adopted above, this equation actually can be derived from equations (11) and (27). It is therefore not an independent equation. 
the dimensionless constant $C_{2}$, which is determined by the microscopic physics of resonant and/or non-resonant coupling between particles and turbulence, is different for protons and electrons. Otherwise, $T_{p} / T_{e}=m_{p} / m_{e}$ and we have

$$
\begin{aligned}
& C_{2}=\frac{\left(5+5 \beta_{t}+4 \beta_{p}\right) H}{9 \beta_{v} l_{e}}\left[\frac{\pi}{2\left(1+\beta_{p}+\beta_{t}\right)}\right]^{1 / 2} \\
& \simeq 0.995 \frac{1+\beta_{t}+0.8 \beta_{p} 0.7 H}{\left(1+\beta_{p}+\beta_{t}\right)^{1 / 2} \beta_{v} l_{e}},
\end{aligned}
$$

If $\beta_{v} \simeq 0.7$ for the MRI as given by Pessah et al. [39, 6, 38 , we will show below that this gives a very inefficient electron heating. Indeed, a higher heating rate will make this non-radiative flow unbound and the accretion will stop. If the particle heating is dominated by the transit-time damping processes, which leads to $T_{p} / T_{e}=m_{p} / m_{e}$ for sufficient heating,

$$
\alpha n k_{\mathrm{B}} T / \tau_{\mathrm{ac}}=\int \Gamma_{k} W(k) \mathrm{d} k=\left\langle\Gamma_{k}\right\rangle B^{2} / 4 \pi,
$$

where $W(k)$ is the turbulence power spectrum and " \langle\rangle " indicates the average over the turbulence power spectrum. In the inertial range, $W(k)=W(k)=u^{2}(H / 2 \pi)^{-1 / 2} k^{-7 / 2}$. Then

$$
C_{2}=\alpha\left(6 l_{e}\left\langle k \cos ^{-1} \theta \sin ^{2} \theta\right\rangle\right)^{-1}
$$

for both electrons and protons if the mean particle velocities are much higher than the phase velocity of the heating waves. The heating will be less efficient for smaller mean particle velocities, leading to higher values of $C_{2}$. In combination with equation (33), one has

$$
\begin{aligned}
& \beta_{v}=\left[\left(5+5 \beta_{t}+4 \beta_{p}\right) H\left\langle k \cos ^{-1} \theta_{\sin ^{2}} \theta\right\rangle / 3 \alpha\right] \\
& {\left[2 \pi /\left(1+\beta_{t}+\beta_{p}\right)\right]^{1 / 2},}
\end{aligned}
$$

This equation shows how the viscous stress is related to the microscopic dissipation processes. For weak turbulence with $\quad \beta_{t} \sim \beta_{p}<<1$,

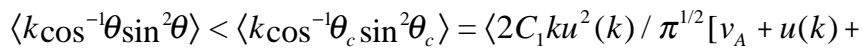
$\left.\left.\left(1+\beta_{p}+\beta_{t}\right) c_{S}^{2} / v_{\mathrm{K}}\right] c_{S}\right\rangle\left\langle\left\langle 2 C_{1} u^{2}(H / 2 \pi)^{-1 / 2} k^{1 / 2} / \pi^{1 / 2}\left[v_{A}+(1+\right.\right.\right.$

$\left.\left.\left.\beta_{p}+\beta_{t}\right) c_{S}^{2} / v_{\mathrm{K}}\right] c_{S}\right\rangle=8 \pi^{1 / 2} C_{1} \beta_{t}\left\{\left(2 \beta_{p}\right)^{1 / 2}+\left(1+\beta_{p}+\beta_{t}\right)[3 /(5+\right.$

$\left.\left.\left.5 \beta_{t}+4 \beta_{p}\right)\right]^{1 / 2}\right\}^{-1} H^{-1}$

. Then one has

$\beta_{v} \leq \frac{80 \pi C_{1} \beta_{t}}{9\left\{\beta_{p}^{1 / 2}+\left(1+\beta_{p}+\beta_{t}\right)\left[3 / 2\left(5+5 \beta_{t}+4 \beta_{p}\right)\right]^{1 / 2}\right\}\left(1+\beta_{t}+\beta_{p}\right)^{1 / 2}}$.

This constraint is similar to equation (21) except now that the flux of released gravitational energy is limited by the TTD processes. Unless there are other energy dissipation mechanisms, the viscosity has to decrease with the decrease of the magnetic field energy density so that the released gravitational energy can be channeled into the internal energy of the gas through the TTD processes.
We note that for the non-radiative accretion flow solution, $\tau_{\mathrm{ac}} \propto r^{3 / 2}$. The turbulence electron heating effectively converts a constant fraction $\delta$ of the viscously dissipated gravitational energy into the internal energy of electrons. The electron heating rate by turbulence is given by $\alpha n k_{\mathrm{B}} T_{e} / \tau_{\mathrm{ac}}$. Then

$$
\delta \simeq\left[\pi\left(5+4 \beta_{p}\right)^{2} / 162 C_{2}^{2} \beta_{v}^{2}\left(1+\beta_{p}\right)\right] m_{e} /\left(m_{p}+m_{e}\right)<1
$$

gives $\quad C_{2} \beta_{v}=1.62 \times 10^{-2}\left(1+0.8 \beta_{p}\right)\left[\delta\left(1+\beta_{p}\right)\right]^{-1 / 2}$. For $\delta=m_{e} /\left(m_{e}+m_{p}\right)$, we reproduce equation (33). Although this result is similar to that proposed in the advection dominated accretion flow models [22], the electron heating studied here becomes very efficient when the electrons become relativistic since the heating time is proportional to the mean speed of electrons.

To make the heating of non-relativistic electrons more efficient than that in the non-radiative flow solution discussed above, one needs a strong magnetic field so that $\beta_{p} \geq 1$ and the mean proton speed is lower than $v_{A}$. The TTD by protons can be reduced significantly for $v_{A}>\left(2 k_{\mathrm{B}} T_{p} / m_{p}\right)^{1 / 2}$ [See eq. (23)]. For $\beta_{p}<1$, it seems that the proton TTD always dominates [20]. One can also increase the electron heating efficiency by reducing its scattering mean free path $l_{e}$. The TTD processes cannot result in strong scattering because they cannot change the perpendicular momentum of these particles. Cyclotron resonances of particles with high frequency waves can change the pitch angle of the particles efficiently. One then needs to take into account the kinetic effects of the plasma waves because the wave frequencies are comparable to the particle gyro-frequencies [51]. However these high frequency waves carry little energy. It appears that cyclotron resonances and the TTD must work coherently to enhance the electron heating rate. A self-consistent modeling of the resonant wave-particle interactions is still under development.

The turbulent motion effectively shares energy and pressure with protons. It therefore only reduces the proton temperature and will not affect the characteristics of emitting electrons significantly. In what follows we will assume that $l_{e}=H$ and explore the characteristic of the accretion model with $\beta_{t} \ll<\beta_{p}=1$, and $C_{2}, \beta_{v}, M$, and $\dot{M}$ as the major model parameters.

For the solution given by equations (17)-(20) and (29)(30), the Coulomb energy exchanges between electrons and protons (with $\tau_{\text {Coul }}$ independent of the radius $r$ ) become relatively more important at larger radii. So we have a onetemperature flow at large radii, which develops into the above solution at the radius, where the Coulomb energy exchange time becomes comparable to the accretion time:

$$
\tau_{\mathrm{vis}} \equiv-r / v_{r} \simeq \frac{\left(5+4 \beta_{p}\right) r^{3 / 2}}{3 \beta_{v} \beta_{p}(G M)^{1 / 2}} .
$$


The transition radius is therefore given by

$$
r_{t} / r_{\mathrm{S}} \simeq 3.39 \times 10^{3}\left(\frac{\beta_{v}^{4} \beta_{p}^{4}\left[1+\beta_{p}\right]}{\left[1+0.8 \beta_{p}\right]^{8}}\right)^{1 / 3}\left(\frac{L_{\mathrm{E} d d}}{\dot{M} c^{2}}\right)^{2 / 3},
$$

where $\quad L_{\mathrm{E} d d}=4 \pi G M\left(m_{p}+m_{e}\right) c / \sigma_{T}$ is the Eddington luminosity and $\sigma_{T}=(8 \pi / 3) e^{4} / m_{e}^{2} c^{4}$ is the Thomson scattering cross-section, and we have assumed a nonradiative accretion with $T_{e}=T_{p}$ at large radii. For a radiative large scale one temperature accretion flow, due to the lower electron temperature, the Coulomb collision time becomes shorter and the transition radius is smaller. We see that twotemperature flows can readily develop at small radii even without considering the electron cooling processes.

When Coulomb collisions become less efficient at smaller radii, in principle the distributions of electrons and protons are not necessarily Maxwellian. However, at a given mean energy, the time scales for electrons and protons reaching equilibrium with themselves are, respectively, $\sim\left(m_{e} / m_{p}\right) \tau_{\text {Coul }}$ and $\sim\left(m_{e} / m_{p}\right)^{1 / 2} \tau_{\text {Coul }}$, which are much shorter than $\tau_{\text {Coul }}$, the time scale for electrons and protons reaching thermal equilibrium with each other [18]. The electron and proton distributions therefore may start to deviate from Maxwellian at $\left(m_{e} / m_{p}\right)^{2 / 3} r_{t}$ and $\left(m_{e} / m_{p}\right)^{1 / 3} r_{t}$, respectively. So the transition radius, where the proton distribution may deviate from Maxwellian, is more than ten times smaller than the radius, where the two-temperature flow starts to develop. The transition radius for electrons is even smaller, and a Maxwellian distribution can be achieved at small radii through relativistic effects and cooling processes [31, 52]. We therefore expect that the electron distribution be always dominated by a thermal component and, over a significant radius range, a two-temperature flow develop self-consistently. The fact that the low-hard state spectra of X-ray binaries can be fitted with the thermal inverse Comptonization model also suggests a dominant thermal electron component.

At very low accretion rates, the Coulomb collision time scales can be much longer than other relevant time scales near the black hole, and we do not expect Maxwellian distributions for both electrons and ions. This is a completely new regime, where the behavior of the turbulent plasma has not been well studied. In these cases, the "temperature" of the particles should be interpreted as a measurement of their mean energies. The actual electron distribution may be constrained by studying its radiation spectrum. We note that the proton distribution is always expected to deviate from Maxwellian at very small radii, where the energy dissipation rate is high, and a high energy proton population may be responsible for the formation of jets in the low states [8, 53]. A detailed investigation of these effects is beyond the scope of this paper.

Since the heating is more efficient when electrons become relativistic, a lower limit of $C_{2}$ can be obtained by considering the accretion processes at small radii. For
$\Lambda=0,\left\langle v_{e}\right\rangle=c$ and $T_{e}=T_{p}, \alpha=3$, the accretion time is given by

$$
\tau_{\mathrm{vis}}=-r / v_{r} \simeq \frac{\left(8+4 \beta_{p}\right) r^{3 / 2}}{3 \beta_{v} \beta_{p}(G M)^{1 / 2}} .
$$

To avoid electrons being overheated, the electron heating time needs to be longer than the accretion time, then we have:

$$
\begin{aligned}
& C_{2} \beta_{v}>\frac{4}{3}\left[\frac{2+\beta_{p}}{3\left(1+\beta_{p}\right)}\right]^{1 / 2}\left(\frac{G M}{r c^{2}}\right)^{1 / 2} . \\
& \text { For } r>10 r_{\mathrm{S}} \text {, we have } \quad C_{2}>0.35\left[\left(1+0.5 \beta_{p}\right) /\right. \\
& \left.\left(1+\beta_{p}\right)\right]^{1 / 2}\left(\beta_{v} / 0.7\right)^{-1} \text {. }
\end{aligned}
$$

For $\Lambda=0$, we solve equations (12) and (27) numerically to constrain the electron heating rate more quantitatively. For $\beta_{p}=1$, we find that $C_{2} \beta_{v}$ needs to be greater than 0.154 to avoid the proton temperature decreasing below zero at small radii at certain accretion rates due to efficient electron heating. For the non-radiative flow solution, $C_{2} \beta_{v}=0.154$ and $\beta_{p}=1$ lead to $T_{p} / T_{e}=55$. Panel "a" of Fig. (1) shows the temperature profiles for $\dot{M}=2 \times 10^{-4}, 0.2,200 L_{\mathrm{E} d d} / c^{2}$. (Note that the Eddington accretion rate is usually defined as $\dot{M}_{\mathrm{E} d d} \equiv 10 L_{\mathrm{E} d d} / c^{2}$.) The other model parameters $C_{2}=0.22$, $\beta_{v}=0.7, \beta_{p}=1$, and $T_{e}=T_{p}=G M m_{p} / 10 k_{B} r_{o}$ at the outer boundary $r_{o}=10^{4} r_{S}$. The disk structure is independent of the black hole mass $M$ because all the relevant length and time scales are proportional to $M$. Equation (39) shows that the Coulomb coupling becomes less efficient with the increase of $\beta_{v}$. For $\beta_{p} \leq 1$, the Coulomb coupling also decreases with the increase of $\beta_{p}$. When $\dot{M}$ is small, Coulomb collision effects are negligible. We recover the solution given by equations (17)-(20) and (29)-(30) at large radii. At small radii, the electron temperature goes beyond that given by equation (29) due to the relativistic effect discussed above. With the increase of $\dot{M}$, the electron and proton temperatures become closer to each other at larger radii. At small radii, because of efficient heating of relativistic electrons, the electron temperature is higher than the proton temperature for $\dot{M}=0.2 L_{\mathrm{E} d d} / c^{2}$. When the accretion rate becomes comparable to or higher than the Eddington accretion rate $\sim 10 L_{\mathrm{E} d d} / c^{2}$, the electron and proton temperatures become identical due to very efficient Coulomb collisions.

The linearly polarized millimeter and sub-millimeter emission from Sagittarius [24, 25, 54] reveals a mass accretion rate below $10^{-5} L_{\mathrm{E} d d} / c^{2}$ [55]. X-ray observations and detailed modeling suggest an even lower accretion rate $[26,28,56]$. VLBI observations have shown that this emission comes from a region within $\sim 15 r_{\mathrm{S}}$ of the black hole and should originate from a hot accretion torus $[27,57]$. The dotted line in Panel "a" of Fig. (1) shows the electron (lower) and proton (upper) temperature profiles for 

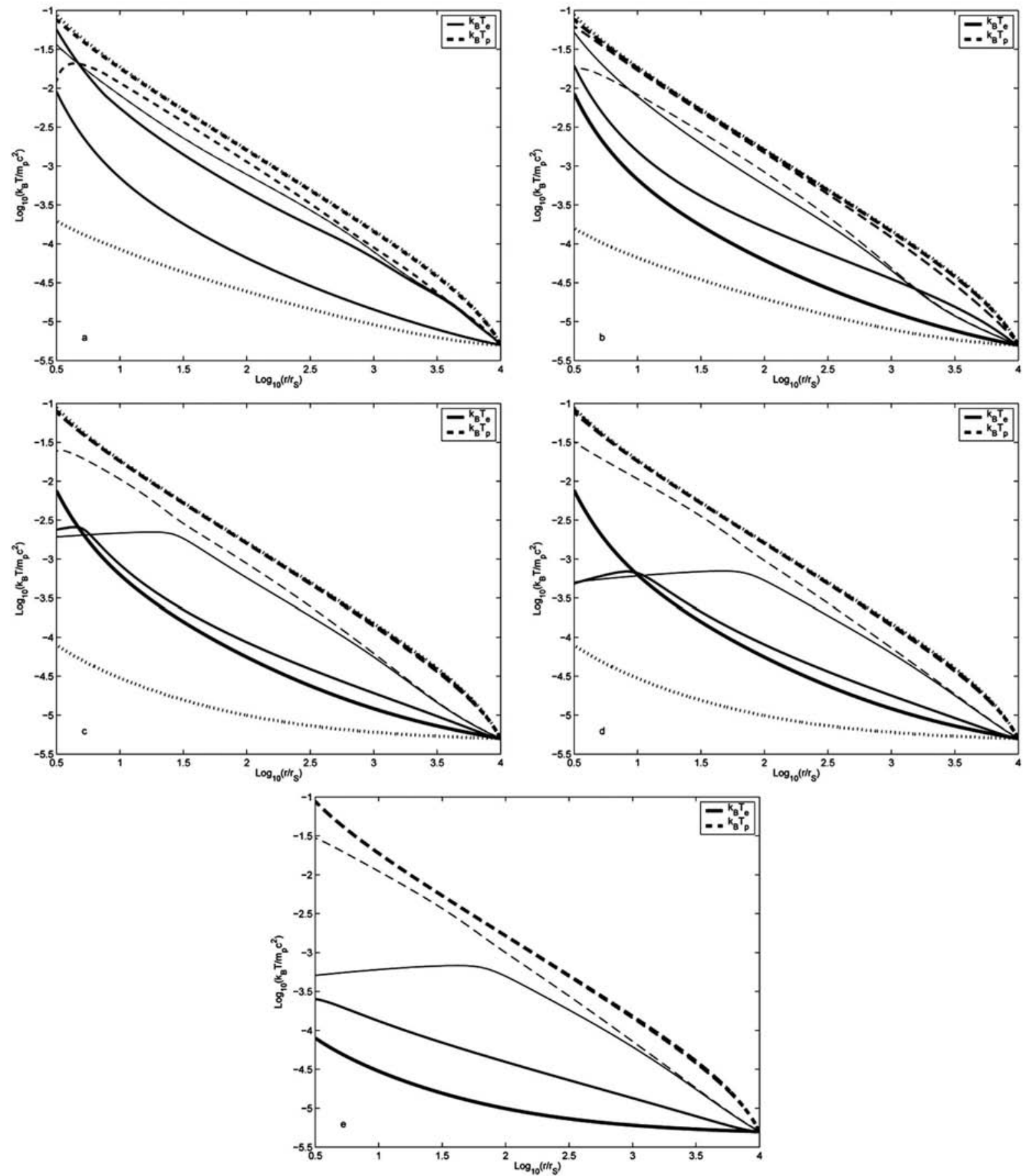

Fig. (1). a: Temperature profiles of electrons (solid) and protons (dashed) for $\dot{M}=2 \times 10^{-4}$ (thick), 0.2 (medium), 200 (thin) $L_{\mathrm{E} d d} / c^{2}$. Cooling is not included to demonstrate the effects of electron heating by turbulence and Coulomb collisions. The electron and proton temperatures at the outer boundary are chosen so that $k_{\mathrm{B}} T_{e}=k_{\mathrm{B}} T_{p}$ is equal to $1 / 10$ of the gravitational binding energy of protons at rest and $\varepsilon<0 . C_{2}=0.22, \beta_{v}=0.7$, and $\beta_{p}=1$. The dotted lines have $C_{2}=1.27$ and $\dot{M}=2 \times 10^{-4} L_{\mathrm{E} d d} / c^{2}$. Note that the proton temperature decreases sharply toward small radii for $\dot{M}=0.2 L_{\mathrm{E} d d} / c^{2}$ and the electron and proton temperatures are identical for $\dot{M}=200 L_{\mathrm{E} d d} / c^{2}$. See text for details. b: Similar to "a" with the bremsstrahlung cooling included. The disk collapses to a cold disk at small radii for $\dot{M}>\dot{M}_{\mathrm{cr}}=1.1 L_{\mathrm{E} d d} / c^{2}\left(r_{o} / 10^{4} r_{\mathrm{S}}\right)^{-1 / 2} . \dot{M}=10^{-4}$ (thick), $10^{-2}$ (medium), 1 (thin) $\dot{M}_{\mathrm{c} r}$. The dotted lines have $C_{2}=1.27$ and the same accretion rate as the thick lines. c: Same as "b" with all the cooling processes included and $M=3.4 \times 10^{6} M_{\odot} \cdot \dot{M}_{\mathrm{cr}}=1.0 L_{\mathrm{E} d d} / c^{2}$. and $\dot{M}=10^{-6}$ (thick), $10^{-3}$ (medium), 1 (thin) $\dot{M}_{\text {cr }}$. We see that the synchrotron and IC coolings only affect the temperature profile of electrons at small radii. d: Same as "c" but for $M=3.4 M_{\odot}$. Because the synchrotron cooling time in the optically thick region scales as $M^{3 / 2}$, the electron temperature decreases with the decrease of $M$ and $\dot{M}_{\mathrm{cr}}=0.72 L_{\mathrm{E} d d} / c^{2}$. e: Same as "d" but for $C_{2}=1.27 . \dot{M}_{\mathrm{cr}}=0.78 L_{\mathrm{E} d d} / c^{2}$ 
$\dot{M}=2 \times 10^{-4} L_{\mathrm{E} d d} / c^{2}$ and $C_{2}=1.27$, which corresponds to equation (33), giving much less efficient electron heating by turbulence. Although we have adopted a relatively high accretion rate, Coulomb collisions are not efficient enough to heat electrons to relativistic energies required to produce the observed synchrotron emission. A fit to the millimeter and sub-millimeter spectrum and polarization gives $C_{2} \beta_{v} \simeq 0.266$ with $\beta_{p}=0.4$, which implies that $T_{p} / T_{e}=115$ for the nonradiative flow solution. The corresponding electron heating rate is comparable to the maximum heating rate derived above with numerical calculations, and is about 4 times higher than that given by equation (33) [58]. One can show that $C_{2}$ for electrons is about 4 times smaller than that for protons in this case. Besides the kinetic effects and the suppression of the TTD by protons with $\beta_{p}=1$, this could also be due to the high mean momentum of protons so that the action of protons on turbulence in their stochastic scattering processes is important. The proton heating is then suppressed giving rise to a $\sim 4$ times longer acceleration time than electrons at a given mean speed and scattering mean free path of both particles.

\section{ELECTRON COOLING}

For a fully ionized magnetized plasma, synchrotron, inverse Comptonization (IC), and bremsstrahlung are the dominated emission mechanisms [59]. In the low accretion states of black holes, the disk is optically thin to bremsstrahlung radiation. We therefore have the bremsstrahlung cooling rate

$$
\Lambda_{\text {brem }}=\left(\frac{2 \pi k_{\mathrm{B}} T_{e}}{3 m_{e}}\right)^{1 / 2} \frac{32 \pi e^{6}}{3 h m_{e} c^{3}} n^{2} g_{\mathrm{B}}=1.4 \times 10^{-27} T_{e}^{1 / 2} n^{2} g_{\mathrm{B}},
$$

where $g_{\mathrm{B}} \simeq 1.2$ is the Gaunt factor and $h$ is the Planck constant. For equations (17)-(20) and (29)-(30), $\Lambda_{\text {brem }} \propto r^{-7 / 2}$ and the bremsstrablung cooling time of electrons $\tau_{\mathrm{bc}}=\alpha k_{\mathrm{B}} n T_{e} / \Lambda_{\mathrm{brem}} \propto r$. Including this energy loss process, we find that the disk collapses to a low temperature flow toward small radii when $\dot{M}>\dot{M}_{\mathrm{cr}}=1.1 L_{\mathrm{E} d d} / c^{2}$ for an outer boundary radius of $10^{4} r_{\mathrm{s}}$. This can be understood by comparing $\tau_{\mathrm{b} c}$ with the accretion time $\tau_{\mathrm{vis}} \propto r^{3 / 2}$ [See eq. (39)]. Then we have

$$
\begin{aligned}
& \dot{M}_{\mathrm{c} r}=\frac{3 \sqrt{3 m_{p} / \pi m_{e}} h \beta_{v}^{2} \beta_{p}^{2} v_{\mathrm{K}}\left(r_{o}\right)}{8 e^{2} g_{\mathrm{B}}}\left(\frac{3}{5+4 \beta_{p}}\right)^{3} \\
& \frac{L_{\mathrm{E} d d}}{c^{2}} \simeq 1.4\left(10^{4} r_{\mathrm{S}} / r_{o}\right)^{1 / 2} \frac{L_{\mathrm{E} d d}}{c^{2}}
\end{aligned}
$$

where we have assumed a non-radiative flow solution with $T_{e}=T_{p}$ at $r_{o}$, and used the model parameters for Panel " a" of Fig. (1) to obtain the last expression. Due to lower outer boundary temperatures used for the numerical calculations, the value of the critical accretion rate is lower than the above analytical estimate. The critical mass accretion rate decreases with the increase of the outer boundary radius.

Panel "b" of Fig. (1) shows the temperature profiles for several values of $\dot{M}$. The other model parameters are the same as Panel "a". Note that the bremsstrahlung cooling has little effect on the temperature profile for $\dot{M} \ll<\dot{M}_{\text {cr }}$ because, compared with the accretion time given by equation (39), this cooling becomes less important toward smaller radii. For $\dot{M}=1.1 \dot{M}_{\mathrm{cr}}$, we see that the temperatures are lowered by the cooling near the outer boundary. Since the cooling rate is proportional to the density squared, the cooling time scale is proportional to $M$. Thus $\dot{M}_{\text {cr }} c^{2} / L_{\text {edd }}$ is independent of $M$, so is the disk structure. Pair production will increase the cooling rate and decrease $\dot{M}_{\mathrm{cr}}$. These results are similar to those given by Narayan \& Yi [60] though electron heating by turbulence has been included in our calculations, implying that the collision-less electron heating does not affect the bremsstrahlung cooling rate at large radii significantly. Since the mean proton energy is lower than $\sim 100 \mathrm{MeV}$, inelastic proton-proton collisions are unimportant for the thermal proton population. However, if there exists a significant high energy non-thermal proton population, strong emission may be produced through this process. The corresponding collision time $\tau_{p p}=1 / \sigma_{p p} n v$, where $\sigma_{p p} \simeq 30 \mathrm{mb}$ is the proton-proton inelastic scattering cross section and $v \sim c$ is the speed of high energy protons. Then we have $\tau_{p p}=\left[\left(5+4 \beta_{p}\right) / 3\right]^{-3 / 2}\left(1+\beta_{p}\right)^{1 / 2} \beta_{v} \beta_{p}\left(\sigma_{T} / \sigma_{p p}\right)\left(r / v_{\mathrm{K}}\right)\left(L_{\mathrm{E} d d} / \dot{M} c^{2}\right)$ $=\left[\left(5+4 \beta_{p}\right) / 3\right]^{-5 / 2}\left(1+\beta_{p}\right)^{1 / 2} \beta_{v}^{2} \beta_{p}^{2}\left(\sigma_{T} / \sigma_{p p}\right)\left(L_{\mathrm{E} d d} / \dot{M} c^{2}\right) \tau_{v i s}$.

For the critical mass accretion rate given by equation (43),

$\tau_{p p}=8 e^{2} g_{\mathrm{B}}\left[\left(1+\beta_{p}\right)\left(5+4 \beta_{p}\right) / 3\right]^{1 / 2} /$ $3 \sqrt{3 m_{p} / \pi m_{e}} h v_{\mathrm{K}}\left(r_{o}\right)\left(\sigma_{T} / \sigma_{p p}\right) \tau_{\mathrm{vis}}=0.70\left(10^{4} r_{S} / r_{o}\right)^{-1 / 2} \tau_{\mathrm{vis}}$.

No strong observational signatures of this process have been identified. We will ignore it in the following.

One may define a critical radius with equation (43):

$$
\begin{aligned}
& r_{\mathrm{c} r} / r_{S}=\frac{27 m_{p} h^{2} \beta_{v}^{4} \beta_{p}^{4} c^{2}}{128 \pi m_{e} e^{4} g_{\mathrm{B}}^{2}}\left(\frac{3}{5+4 \beta_{p}}\right)^{6}\left(\frac{L_{\mathrm{Edd}}}{\dot{M} c^{2}}\right)^{2} \\
& \simeq 2.0 \times 10^{4}\left(\frac{L_{\mathrm{Edd}}}{\dot{M} c^{2}}\right)^{2} .
\end{aligned}
$$

A hot accretion flow can only exist below $r_{\mathrm{cr}}$. When the transition radius $r_{t}$ given by equation (40) is greater than $r_{\mathrm{cr}}$, as is the case for relatively high accretion rates, a hot twotemperature flow may develop below $r_{c r}$. For low accretion rates, $r_{\text {cr }}$ can be much greater than $r_{t}$, we have a hot onetemperature flow between $r_{t}$ and $r_{\text {cr }}$, which develops into a hot two-temperature flow below $r_{t}$. The thin lines in Panel "b" corresponds to this case, where $r_{t} \sim 1600 r_{S}$ 
and $\quad r_{\mathrm{c} r}=10^{4} r_{S}$. From $r_{t}=r_{\mathrm{c} r}$, we have $\dot{M} c^{2} / L_{\mathrm{E} d d} \simeq 156 \beta_{v}^{2} \beta_{p}^{2} /\left(1+\beta_{p}\right)^{1 / 4}\left(1+0.8 \beta_{p}\right)^{5 / 2} \quad$ and $r_{t}=r_{\mathrm{c} r} \simeq 117 r_{S}\left(1+\beta_{p}\right)^{1 / 2} /\left(1+0.8 \beta_{p}\right)$. However, how exactly the hot flow may develop toward small radii is still an open question, which may depend on the nature of the large-scale flow and the coupling between the disk and its corona [61].

When the source is optically thin to synchrotron radiation, the cooling rate is given by

$$
\Lambda_{\mathrm{syn}}=\frac{4 e^{4} n}{9 m_{e}^{4} c^{5}}\left\langle p^{2}\right\rangle B^{2} \simeq 1.06 \times 10^{-15} n B^{2} \frac{3 x^{2}+12 x+12}{x^{3}+x^{2}},
$$

where $\left\langle p^{2}\right\rangle$ is the mean momentum square of the electrons [62]. For equations (17)-(20) and (29)-(30) and $x<<1$, $\Lambda_{\mathrm{syn}} \propto r^{-6}$. The corresponding cooling time scale $\tau_{\mathrm{sc}} \propto r^{7 / 2}$, which is more important at smaller radii. For $x>>1$, $\Lambda_{\mathrm{s} y n} \propto r^{-5}$ and $\tau_{\mathrm{sc}} \propto r^{5 / 2}$. However, most of the thermal synchrotron emission is emitted at

$$
\begin{aligned}
& v_{E} \simeq \frac{x+20}{x+1} v_{c}=\frac{60(1+0.05 x) e B \gamma_{c}^{2}}{4 \pi(x+1) m_{e} c}= \\
& 8.4 \times 10^{7} B \gamma_{c}^{2}(1+0.05 x) /(x+1) \mathrm{H} z,
\end{aligned}
$$

where $\gamma_{c}=1 / x+1$ [50]. The thermal synchrotron emission (emissivity per solid angle) and absorption coefficients are given, respectively, by $[63,64]$

$$
\begin{gathered}
\mathscr{E}_{v}=\frac{\sqrt{3} e^{3}\left\langle p^{2}\right\rangle(x+1)}{8 \pi m_{e}^{3} c^{4}\left\langle\gamma^{2}\right\rangle(1+0.19 x)} B n z_{M} I\left(z_{M}\right), \\
\kappa_{v}=\frac{\mathscr{E}_{v} c^{2}\left[\exp \left(h v / k_{\mathrm{B}} T_{e}\right)-1\right]}{2 h v^{3}} \simeq \frac{\pi e n\left\langle p^{2}\right\rangle}{3 \sqrt{3} \gamma_{c}^{4} m_{e}\left\langle\gamma^{2}\right\rangle B k_{\mathrm{B}} T_{e}} \\
\frac{I\left(z_{M}\right)(x+1)}{z_{M}(1+0.19 x)}
\end{gathered}
$$

where $\left\langle\gamma^{2}\right\rangle \simeq(x+12)(x+1) / x^{2}$ is the mean Lorentz factor square of electrons, $(x+1) /(1+0.19 x)$ is chosen to take into account the non-relativistic effects (see below), and

$$
\begin{gathered}
I\left(z_{M}\right)=4.0505 z_{M}^{-1 / 6}\left(1+0.40 z_{M}^{-1 / 4}+0.5316 z_{M}^{-1 / 2}\right) \\
\exp \left(-1.8899 z_{M}^{1 / 3}\right), \\
z_{M}=v / v_{c} \equiv 4 \pi m_{e} c v / 3 e B \gamma_{c}^{2} .
\end{gathered}
$$

We then have the optical depth through the emission region

$$
\tau_{v}(v) \equiv \kappa_{v}(v) H=\frac{\pi e\left\langle p^{2}\right\rangle n H}{3 \sqrt{3} m_{e}\left\langle\gamma^{2}\right\rangle k_{\mathrm{B}} T_{e} \gamma_{c}^{4} B} \frac{I\left(z_{M}\right)(x+1)}{z_{M}(1+0.19 x)},
$$

which, for a given $z_{M}$, is proportional to $r^{23 / 4}$ for equations (17)-(20) and (29)-(30) and $x<<1$ and proportional to $r^{3 / 4}$ for $x>>1$. When $\tau_{v}\left(v_{E}\right)$ becomes greater than unity at large radii, one has to take into account the self-absorption effects. The corresponding synchrotron cooling rate may be approximated as

$$
\begin{aligned}
& \Lambda_{\mathrm{syn}}=\frac{16 \sqrt{3} \pi^{2} k_{\mathrm{B}} T_{e} v_{E}^{3}}{45^{2} I[(x+20) /(x+1)] c^{2} H} \frac{\left\langle\gamma^{2}\right\rangle(1+x)(1+0.19 x)}{\gamma_{c}^{2}(1+0.05 x)^{2}} \\
& =\frac{80 \sqrt{3} e^{3} k_{\mathrm{B}}}{3 \pi I[(x+20) /(x+1)] m_{e}^{3} c^{5}} \frac{T_{e} B^{3}\left\langle\gamma^{2}\right\rangle \gamma_{c}^{4}(1+0.05 x)(1+0.19 x)}{H(x+1)^{2}} \\
& \simeq 6.4 \times 10^{-13} \frac{T_{e} B^{3}}{H} \frac{(x+12)(x+1)^{3}(1+0.05 x)(1+0.19 x) I(20)}{x^{6} I[(x+20) /(x+1)]},
\end{aligned}
$$

where the numerical factor is chosen so that the cooling rates given by equations (45) and (52) are equal when $\tau_{v}\left(v_{E}\right)=1$, and $\Lambda_{\mathrm{syn}} \simeq 8 \pi k_{\mathrm{B}} T_{e} v_{E}^{3} / 3 c^{2} H$ for $x \gg>1$. That is, for cyclotron radiation in the optically thick regime, we assume a black body spectrum cutting off at $v_{E}=v_{c}$. This cooling is important at small radii since $\Lambda_{\mathrm{syn}} \propto r^{-47 / 4}$ and $\tau_{\mathrm{sc}} \propto r^{37 / 4}$ for equations (17)-(20) and (29)-(30) and $x<<1$. For $x>>1$, $\Lambda_{\mathrm{syn}} \propto r^{-23 / 4}$ and $\tau_{\mathrm{sc}} \propto r^{13 / 4}$.

We will assume that the IC is in the Thomson limit and the seed photons for IC are provided by the synchrotron radiation. Then we have the total cooling rate

$$
\begin{aligned}
& \Lambda=\Lambda_{\mathrm{syn}}+\Lambda_{\mathrm{IC}}+\Lambda_{\mathrm{brem}}=\Lambda_{\mathrm{syn}}\left[1+8 \pi\left(\Lambda-\Lambda_{\mathrm{brem}}\right) H / c B^{2}\right]+\Lambda_{\mathrm{brem}} \\
& =\Lambda_{\mathrm{syn}}\left(1-8 \pi \Lambda_{\mathrm{syn}} H / c B^{2}\right)^{-1}+\Lambda_{\mathrm{brem}} .
\end{aligned}
$$

Panel "c" of Fig. (1) shows the temperature profiles with the above cooling processes included for $M=3.4 \times 10^{6} M_{\odot}$. The critical mass accretion rate $\dot{M}_{\mathrm{cr}}=1.0 L_{\mathrm{E} d d} / c^{2}$ is slightly lower that in Panel "b", where only the bremsstrahlung cooling is considered. This is consistent with the fact that the synchrotron and IC coolings are unimportant at large radii. The model parameters are the same as Panel " $b$ " except $\dot{M}=10^{-6}$ (thick), $10^{-3}$ (medium), 1.0 (thin) $\dot{M}_{\text {cr }}$. We note that the electron temperature never goes above $\sim 10^{11} \mathrm{~K}$, which may provide an alternative explanation to the observed upper limit in brightness temperature of powerful extragalactic radio sources [65]. At small radii, these cooling processes do reduce the electron temperature.

For the non-radiative accretion flow solution given by equations (17)-(20) and (29)-(30) with $T_{e}=T_{p}, x<<1$ at small radii. The synchrotron emission can be optically thin and therefore polarized at

$$
\begin{aligned}
& v_{c}=\frac{3 e B\left(k_{\mathrm{B}} T_{e}\right)^{2}}{4 \pi m_{e}^{3} c^{5}}=\frac{3^{3 / 2}\left(m_{e}+m_{p}\right)^{5 / 2} c^{3}}{2^{29 / 4} e m_{e}^{2}\left(G M \beta_{v}\right)^{1 / 2}\left(1+\beta_{p}\right)^{1 / 4}} \\
& \left(\frac{3}{5+4 \beta_{p}}\right)^{7 / 4}\left(\frac{r}{r_{S}}\right)^{-13 / 4}\left(\frac{\dot{M} c^{2}}{L_{\mathrm{Edd}}}\right)^{1 / 2} \\
& \simeq 2.9 \times 10^{14} \beta_{v}^{-1 / 2}\left(1+\beta_{p}\right)^{-1 / 4}\left(\frac{9}{5+4 \beta_{p}}\right)^{7 / 4}\left(\frac{r}{3 r_{S}}\right)^{-13 / 4}
\end{aligned}
$$

$\left(10^{8} M \dot{M} c^{2} M L_{\mathrm{E} d d}\right)^{1 / 2} \mathrm{H} z$ 


$$
\begin{aligned}
& \tau_{v}\left(v_{c}\right) \sim \frac{1.18 \pi e m_{e}^{5} c^{10} n H}{3 \sqrt{3}\left(k_{\mathrm{B}} T_{e}\right)^{5} B}=660 \beta_{p}^{-1}\left(1+\beta_{p}\right)^{1 / 4} \\
& \left(\frac{9}{5+4 \beta_{p}}\right)^{-23 / 4}\left(\frac{r}{3 r_{S}}\right)^{23 / 4}\left(\frac{M \dot{M} c^{2}}{10^{8} M \cdot L_{\mathrm{Edd}}}\right)^{1 / 2}
\end{aligned}
$$

The synchrotron cooling does not affect the temperature profile significantly when $\tau_{v}\left(v_{c}\right)<1$. This is the case for $\dot{M}=10^{-6} \dot{M}_{\text {cr }}$. With the increase of $\dot{M}$, the synchrotron cooling rate increases much faster than the electron heating rate. The disk becomes optically thick to synchrotron emission, which dominates the cooling at small radii. For relativistic electrons with $x<<1$, the synchrotron cooling time $\tau_{\mathrm{sc}} \propto n H / B^{3} T_{e}^{6} \propto r^{13 / 4} T_{e}^{-6}$. The electron heating time $\tau_{\mathrm{ac}} \propto H / v_{\mathrm{A}}^{2} \propto r^{2}$. The balance of the two gives $T_{e} \propto r^{5 / 24}$, which explains why the electron temperature increases slowly with radius at small radii for $\dot{M}=10^{-3} \dot{M}_{\text {cr }}$. Coulomb collisional heating of electrons dominates close to the critical accretion rate. The corresponding heating time is proportional to $\tau_{\mathrm{Coul}} T_{e} / T_{p} \propto r^{5 / 2} T_{e}$. The balancing of the optically thick synchrotron cooling with Coulomb collisional heating leads to $T_{e} \propto r^{3 / 28}$, which explains the very slow increase of the electron temperature with radii at small radii for $\dot{M}=\dot{M}_{\mathrm{cr}}$.

Because the synchrotron cooling rate in the optically thick regime is proportional to $n^{3 / 2} / H$, the cooling time is proportional to $M^{3 / 2}$. This cooling becomes relatively more efficient for smaller black holes so that the electron temperature decreases with the decrease of $M$ for given $\dot{M} c^{2} / L_{\mathrm{E} d d} \cdot \dot{M}_{\text {cr }}$ also decreases slightly with the decrease of $M$. For $M=3.4 M_{\odot}$, we find $\dot{M}_{\mathrm{cr}}=0.72 L_{\mathrm{E} d d} / c^{2}$. Panel "d" of Fig. (1) is the same as Panel "c" except that $M=3.4 M_{\odot}$. We therefore expect that, for $\dot{M}=\dot{M}_{\mathrm{cr}}$, the intrinsic brightness temperatures for extragalactic radio sources are higher for bigger black holes and there should be a correlation between the brightness temperature and the source luminosity. Pair production effects need to be incorporated to give more quantitative predictions. Due to the low radiation efficiency at large radii, the critical mass accretion rate is not very sensitive to the electron heating rate either. Panel "e" of Fig. (1) show the temperature profiles for $C_{2}=1.27$. The other parameters are the same as Panel "d", and $\dot{M}_{\mathrm{cr}}=0.78 L_{\mathrm{E} d d} / c^{2}$.

\section{APPLICATION TO X-RAY BINARIES IN THE LOW-HARD STATES}

It has been suggested that Coulomb collisions are efficient enough to heat electrons to explain observations of galactic X-ray binaries in the low-hard states [14, 22]. Although some of the relevant models may achieve an electron temperature in line with observations by adjusting parameters describing the source structure and/or dissipation processes, the related assumptions are not well justified both theoretically and observationally. It is also not obvious whether these models can explain the recently observed anticorrelation between the electron temperature and the Thomson optical depth of Cygnus X-1 over a large dynamical range [32]. The model proposed here is selfconsistent and has the basic parameters to describe the dynamics and radiative processes. It is therefore well positioned to uncover new physical processes when applied to specific observations.

With the parameters adopted above, the model clearly cannot explain the high hard X-ray luminosities observed in some of the galactic X-ray binaries. The hard X-ray luminosity can be a significant fraction of $L_{\mathrm{E} d d}$ for some sources. A much higher $\dot{M}_{\mathrm{cr}}$ is needed to produce such a high luminosity. To increase $\dot{M}_{\mathrm{cr}}$, one may increase the viscosity by increasing $\beta_{v}$ or decrease the outer boundary radius. The virial temperature at $r=10^{4} r_{\mathrm{s}}$ is already a few tens of $\mathrm{keV}$, and we are interested in showing how electrons reach this temperature range from a large-scale cool accretion flow. We will consider the first option and set the outer boundary temperature $k_{\mathrm{B}} T_{e}=k_{\mathrm{B}} T_{p}=5 \times 10^{-6} m_{p} c^{2} \sim 5$ $\mathrm{keV}$ in the following. Panel "a" of Fig. (2) shows the temperature profiles for $M=3.4 M_{\odot}, C_{2}=0.2, \beta_{p}=1$, and $\beta_{v}=3$, which imply that $T_{p}=846 T_{e}$ for the non-radiative solution. The critical mass accretion rate $\dot{M}_{\mathrm{cr}}=18.2 L_{\mathrm{E} d d} / c^{2}$, which is slightly higher than that given by equation (43) due to the high value of $\beta_{v}$ that makes the radial velocity significant. Therefore, if the hot accretion flow develops naturally with the processes considered here, a very high viscosity is needed to produce a hard X-ray luminosity on the order of $0.1 L_{\mathrm{E} d d}$. This statement is not necessarily true if the formation of hot accretion flows is triggered by some instability near the black hole.

However, for $\dot{M} \sim \dot{M}_{\mathrm{cr}}$, the electron temperature is more than $1 \mathrm{MeV}$ near the black hole, which is much higher than the observed values in the low-hard states (See Fig. 2a). It is clear that, besides the local internal cooling processes considered above, other cooling processes have to be introduced to bring the electron temperature to the desired energy range. Observations of X-ray binaries in the low-hard states show that photons from the large-scale optically thick slim disk can provide sufficient soft photons to cool electrons in the inner two-temperature flow through IC [66]. [66]. The cooling rate will also increase if we consider a global radiation transfer and include the cooling due to pair production. Here we treat these cooling processes approximately by assuming the energy density of the soft photons produced outside the two-temperature accretion flow is three times higher than the local magnetic field energy density so that there is an extra cooling term 3 times higher than that given by equation (45). Panel "b" of Fig. (2) shows the temperature profiles with this cooling effect included. In this case, $\dot{M}_{\mathrm{c} r}=0.741 L_{\mathrm{E} d d} / c^{2}$, which is clearly affected by this extra cooling process. The other model parameters are the same as Panel "a". 
Because the cooling is dominated by the IC processes, the corresponding cooling time $\tau_{\mathrm{IC}} \propto 1 / B^{2} \propto r^{5 / 2}$. The electron heating times through the collision-less and collisional processes are proportional to $T_{e}^{1 / 2} r^{2}$ and $T_{e}^{5 / 2} r^{5 / 2}$, respectively. The latter dominates when $\dot{M}$ is close to $\dot{M}_{\text {cr }}$ and at small radii, and one has an electron temperature independent of the radius. The value of the electron temperature depends on the ratio of the external photon energy density to that of the magnetic field $\eta$ :

$$
k_{\mathrm{B}} T_{e} \simeq\left(\frac{9 \ln \lambda m_{e}}{4 \sqrt{2 \pi} \beta_{p} \eta m_{p}}\right)^{2 / 5} m_{e} c^{2} .
$$

The electron heating by turbulence becomes relatively more important for lower values of $\dot{M}$. However, with $\dot{M}$, which is proportional to the Thomson optical depth of the
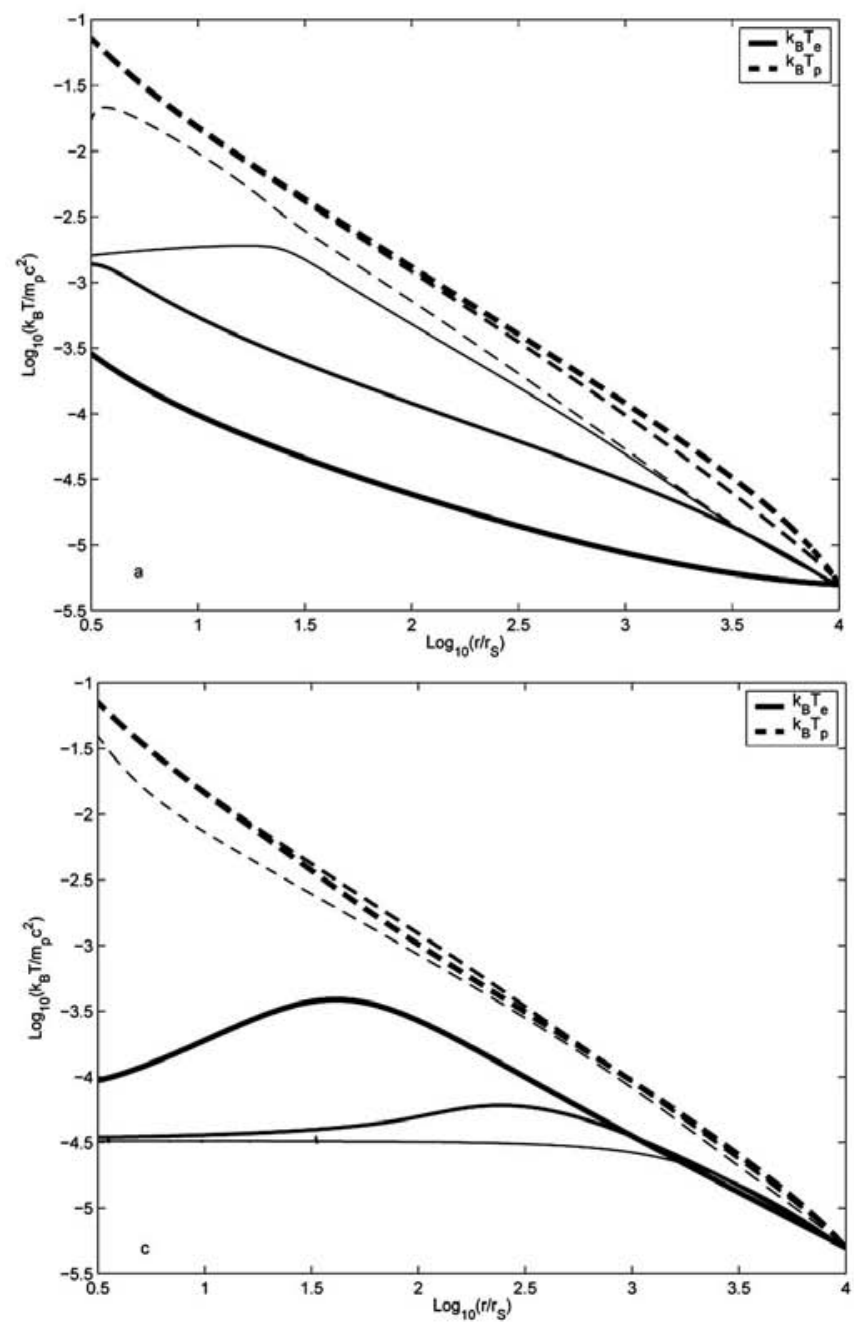

accretion flow, varying by two orders of magnitude, the electron temperature in the inner region remains about 30 $\mathrm{keV}$. This is not consistent with the recently observed anticorrelation between the electron temperature and the Thomson optical depth of Cygnus X-1 in the low-hard and intermediate states [32, 67].

By increasing the electron heating rate by one order of magnitude, i.e. with $C_{2}=0.02$ and $T_{p}=7.5 T_{e}$ for the nonradiative solution, we obtain temperature profiles in Panel "c". Due to the dominance of this collision-less electron heating for $\dot{M}=10^{-2} \dot{M}_{\mathrm{cr}}$, one has $T_{e} \propto r$. For $\dot{M}=10^{-1} \dot{M}_{\text {cr }}$, Coulomb collisional heating dominates at small radii and the collision-less heating becomes more important with the increase of $r$. At even large radii, the cooling is unimportant. These explain the electron temperature profile. The other model parameters are the
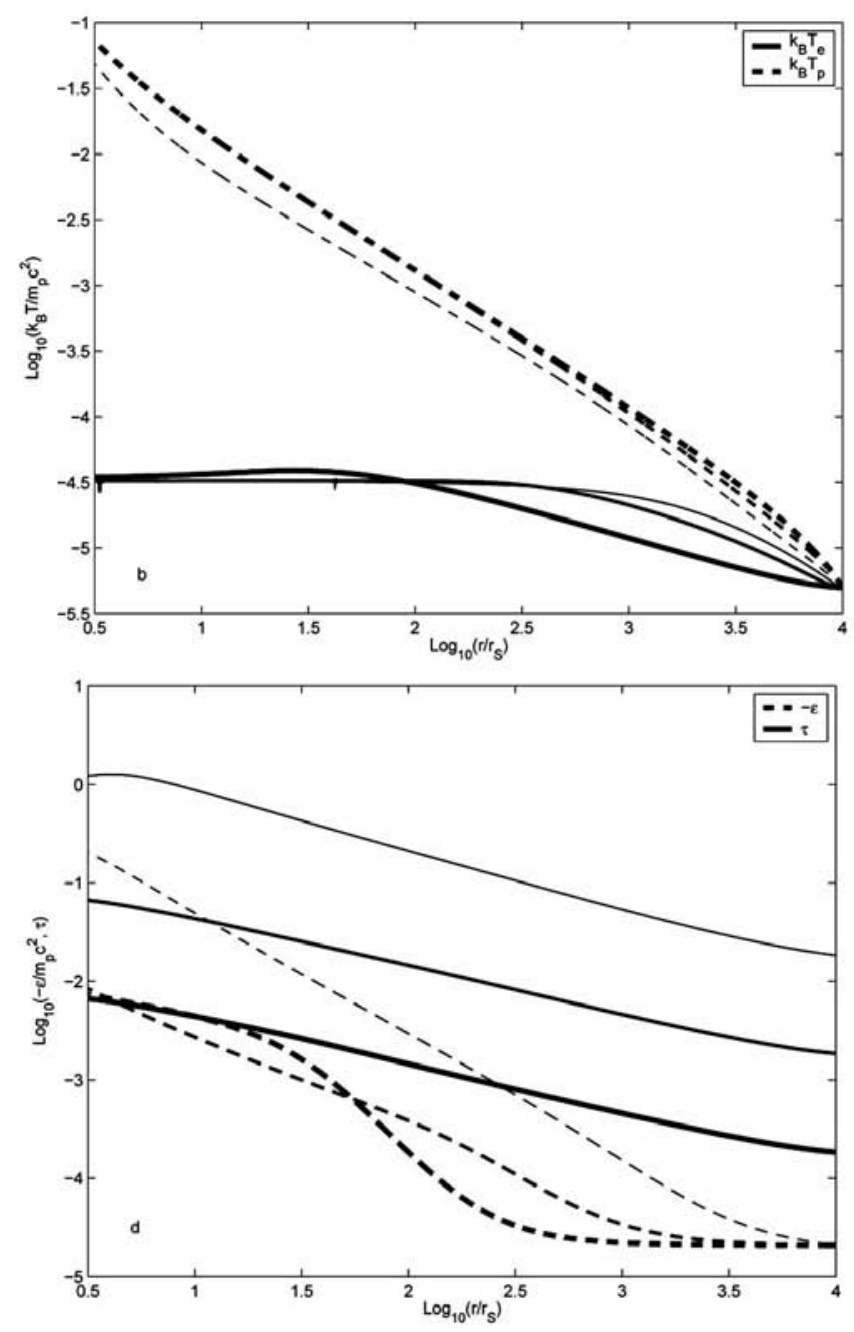

Fig. (2). a: Same as "e" of Fig. (1) but for $M=34 M_{\odot}, C_{2}=0.2, \beta_{p}=1, \beta_{v}=3$. In this case, $\dot{M}_{\mathrm{cr}}=18.2 L_{\mathrm{E} d d} / c^{2} . \dot{M}=10^{-4}$ (thick), 0.01 (medium), 1.0 (thick) $\dot{M}_{\mathrm{cr}}$. b: Same as "a" but with an external soft photo field, whose energy density is 3 times higher than the local magnetic field energy density. $\dot{M}_{\mathrm{cr}}=0.741 L_{\mathrm{E} d d} / c^{2}$, and $\dot{M}=10^{-2}$ (thick), 0.1 (medium), 1.0 (thin) $\dot{M}_{\mathrm{cr}}$. c: Same as "b" but for $C_{2}=0.02$. d: Profiles of $\varepsilon$ (dashed) and $\tau$ (solid) for the three models in "c". The thickness of the lines are the same for a given accretion rate. The luminosity of the disk for the thin lines is more than $9 \%$ of $L_{\mathrm{E} d d}$. 
same as Panel "b". We see that the electron temperatures are in the observed energy range. Panel "d" shows profiles of the Thomson optical depth $\tau=2 \sigma_{T} H n$ and $\varepsilon$ for the same models in Panel "c". We qualitatively recover the observed anti-correlation between $k_{\mathrm{B}} T_{e}$ and $\tau$. The radiation efficiency of the accretion flow is $\sim 0.01$ giving rise to a luminosity of $\sim 10^{35-38} \mathrm{erg} \mathrm{s}^{-1}$, which is also in line with observations.

\section{CONCLUSIONS AND DISCUSSION}

To explain the millimeter and sub-millimeter spectrum and polarization of Sagittarius A* and the recently observed anti-correlation between the electron temperature and the Thomson optical depth of Cygnus X-1 in the low-hard and intermediate states, we show that electrons need to be heated efficiently by turbulence plasma waves. Coulomb collisions are not effective enough to heat electrons to the temperature required by observations of Sagittarius $A^{*}$ that has a very low accretion rate.

We first consider the energy flow in the accretion system. Due to the shearing of the Keplerian two temperature accretion flow, the turbulence cascade from large to small scales is suppressed. Unless direct heating of plasmas by large scale turbulence is significant, the viscous stress needs to be proportional to the product of the turbulence kinetic energy density and the total turbulence energy density to balance the viscous gravitational energy dissipation and the turbulence energy cascade. This can be verified with shearing box numerical simulations and may not be consistent with the suggestion that the viscous stress is proportional to the magnetic field energy density and the Maxwell stress always dominates [39].

If the collision-less heating is dominated by the TTD processes, the ratio of the magnetic field energy density needs to be comparable to the thermal energy density of the plasma to suppress the proton heating rate. Otherwise, the proton TTD dominates and little electron heating is expected. The electron heating may also be enhanced due to the resonant scattering of particles by high frequency waves. Such scattering can effectively reduce the particle scattering mean free path and increase the heating rate through stochastic wave particle interactions. A detailed modeling of the turbulence cascade and coupling with plasma waves and background particles is needed to obtain more quantitative results. Since the electron heating rate is proportional to the mean electron momentum and the Alfvén velocity squared, the heating of relativistic electrons can be very efficient, and efficient electron heating may also be realized through strong magnetized coronas. However, the coronas need to be energetically significant in this case.

The critical mass accretion rate, below which a twotemperature solution may exist, is determined by the radiative cooling and Coulomb collisional heating processes and is almost independent of the collision-less electron heating by turbulence. To reproduce the observed high luminosity of X-ray binaries in the low accretion states, a high viscosity is required to increase the critical mass accretion rate, and the radial velocity of the accretion flow can be comparable to the Keplerian velocity. We show that the electron cooling for galactic X-ray binaries needs to be dominated by IC of an external soft photon field originated presumably from a large-scale cold slim disk. Synchrotron, synchrotron self-Comptonization, and bremsstrahlung processes inside the hot accretion flows are not efficient enough to cool the electrons to the observed temperature range. Assuming that the energy density of these external photons is 3 times higher than the local magnetic field energy density, we find that the electron temperature at the critical accretion rate is determined by this energy density ratio. A lower external photon energy density gives rise to a higher electron temperature. This high external photo energy density suggests that the radiation field may have important dynamical effects and may be partially responsible for the required high viscosity.

Recent observations of Cygnus X-1 show that the electron temperature in the IC model [67] decreases by more than a factor of 10 when the Thomson optical depth, which is proportional to the accretion rate, increases by two orders of magnitude in the low-hard and intermediate states. We show that very efficient electron heating by turbulence can reproduce such an anti-correlation. Without this extra heating process (besides the Coulomb collisional heating), the electron temperature is almost independent of the accretion rate. It appears that such an anti-correlation may also be explained by adjusting the properties of the external photo field [22]. It is, however, not clear whether the observed dramatic changes in both the electron temperature and the Thomson optical depth can be reproduced in such a scenario. Detailed modeling of the external photo field is required to address this issue and to fit the broadband spectrum of Cygnus X-1 in the low-hard and intermediate states.

We have shown that a two-temperature flow can develop self-consistently at small radii for low accretion rates mostly due to the fact that electrons and protons reach a thermal equilibrium with themselves much faster than with each other through Coulomb collisions. However, at very low accretion rates, there is no guarantee that the proton distribution is Maxwellian although electrons may reach a quasi-thermal distribution due to balancing between the cooling and heating processes. Observations of the low-hard states of X-ray binaries also suggest that the electron distribution is dominated by a thermal component. When Coulomb collision time scales are much longer than other relevant time scales, the particle "temperature" should be interpreted as a measurement of the internal energy but not a thermal distribution.

With the pseudo-Newtonian potential and assumption of Keplerian azimuthal velocities, processes below $\sim 3 r_{\mathrm{s}}$ can not be described accurately by our model. These processes can have important observational consequences [17] and may drive strong outflows responsible for the observed radio emission $[10,12,68]$. By dropping these assumptions and considering the momentum conservation in the radial direction, one may build a more complete model to study these processes [69]. 
In the model we have ignored the vertical structure of the disk, dynamical effects of the radiation field, winds and/or outflows, outwardly directed waves, and pair productions, which can be important for X-ray binaries. These may introduce a factor of a few changes in our model parameters, especially in regards to the temperature profile of protons and the transition from an one-temperature flow at large radii to the two-temperature inner region, which may have important implications on the origin of quasi-periodic oscillations. But our conclusion of the presence of efficient collision-less electron heating by turbulence is not affected. Electrons can also be accelerated by MHD turbulence to very high energies in coronas of the disk and produce the observed non-thermal high energy spectral component [3]. These electrons carry much less energy than the thermal electron population considered in this paper and can be treated with appropriate corona models $[8,9]$.

Our treatment of the electron heating by turbulence is different from that proposed in the advection dominated accretion flow models [23], where the energy equation is separated into two equations, one for electrons and the other for ions, and it is assumed that a fixed fraction of the viscously dissipated energy is deposited into electrons. We point out that equation (11) is the more appropriate energy equation and there is no reasonable justification to split it into two equations except that the coupling between electrons and ions through plasma waves or turbulence can be ignored. Our results and the MRI clearly show the opposite, i.e. turbulence plays crucial roles in an accretion flow. It is also difficult to understand why a fixed fraction of the viscously dissipated energy goes into electrons. Electron heating by plasma waves should depend on the properties of the plasma and turbulence $[19,20]$. Equation (27) is perhaps more appropriate to describe the temperature evolution of electrons.

It is very challenging to study turbulence, one of the commonest natural phenomena. It originates from the nonlinear nature of complex many body systems. In astrophysics, MHD turbulence is ubiquitous, and one of the most important effects of it is the energization of charged particles in collision-less plasmas. The microscopic details of this stochastic particle acceleration process are still a matter of debate. Even the common practice of treating MHD turbulence as a spectrum of plasma waves is not well justified, especially for strong turbulence. The results in this paper provide a means to study the energization of electrons and ions by turbulence, specifically the energy partition between the two particles, with observations. Our results suggest that the electron heating time by turbulence is at least 4 times shorter than that of protons at a given mean particle speed. Detailed modeling of the radiation spectrum and MHD simulations of the accretion flows will lead to more quantitative results.

We note that the dimensionless parameter $C_{2}$, which characterizes the electron heating by turbulence in our model, decreases by a factor of $>10$ from the supermassive black hole of Sagittarius A* to the stellar mass black hole in Cygnus X-1. This may be related to the dramatic increase of the turbulence viscosity described by $\beta_{v}$, which changes from 0.7 for Sagittarius $A^{*}$ to 3 for galactic X-ray binaries. Indeed, the product of the two changes by less than a factor of 3 .

\section{ACKNOWLEDGEMENTS}

This work is supported by a start-up grant at the Purple Mountain Observatory, the EU's SOLAIRE research and Trainting Network at the University of Glasgow (MTRNCT-2006-035484), and, under the auspices of the U.S. Dept. of Energy, supported by its contract W-7405-ENG-36 to the Los Alamos National Laboratory. Some ideas of the paper were conceived while SL was visiting TIARA in 2007. SL thanks Dr. Ron Taam and Dr. Feng Yuan for their hospitalities and Dr. Xue-bing Wu and Lei Qian for useful discussions.

\section{REFERENCES}

[1] Shakura NI, Sunyaev RA. Black holes in binary systems. Observational appearance. Astron Astrophysc 1973; 24: 337.

[2] Gierlinski M, Done C. Is the soft excess in active galactic nuclei real? MNRAS 2004; 349: 7 .

[3] Gierlinski M, Zdziarski AA, Poutanen J, Coppi PS, Ebisawa K, Johnson WN. Radiation mechanisms and geometry of Cygnus X-1 in the soft state. MNRAS 1999; 309: 496.

[4] Zhang SN, Cui W, Chen W, Yao Y, et al. Three-Layered Atmospheric Structure in Accretion Disks Around Stellar-Mass Black Holes. Science 2000; 287: 1239.

[5] Chandrasekhar S. Hydrodynamic and hydromagnetic stability. Chap. 9, Dover, Publ. Inc. 1961.

[6] Ichimaru S. Bimodal behavior of accretion disks: theory and application to Cygnus X-1 transitions. Astrophysc J 1977; 214: 840 .

[7] Balbus SA, Hawley JF. A powerful local shear instability in weakly magnetized disks. II. nonlinear evolution. Astrophysc J 1991; 555: L83.

[8] Dermer CD, Miller JA, Li H. Stochastic Particle Acceleration near Accreting Black Holes. Astrophysc J 1996; 456: 106.

[9] $\mathrm{Li} \mathrm{H}$, Miller JA. Electron acceleration and the production of nonthermal electron distributions in accretion disk coronae. Astrophysc J 1997; 478: L67.

[10] Fender RP, Belloni TM, Gallo E. Towards a unified model for black hole X-ray binary jets. MNRAS 2004; 355: 1105.

[11] Remillard RA, Morgan EH, McClintock JE, Bailyn CD, Orosz JA RXTE observations of 0.1-300 HZ quasi-periodic oscillationsin the microquasar GRO J1655-40. Astrophysc J 1999; 522: 397.

[12] Falcke H, Körding E, Markoff S. A scheme to unify low-power accreting black holes. Jet-dominated accretion flows and the radio/X-ray correlation . Astron Astrophysc 2004; 414: 895.

[13] Camenzind M. Relativistic outflows from active galactic nuclei. MmSAI 2005; 76: 98.

[14] Shapiro SL, Lightman AP, Eardley DM. A two-temperature accretion disk model for Cygnus X-1 - Structure and spectrum. Astrophysc J 1976; 204: 187.

[15] Rees MJ, Begelman MC, Blandford RD, Phinney ES. Ionsupported tori and the origin of radio jets. Nature 1982; 295: 17

[16] Zdziarski AA, Poutanen J, Paciesas WS, Wen LQ. Understanding the long-term spectral variability of cygnus $\mathrm{x}-1$ with burst and transient source experiment and all-sky monitor observations. Astrophysc J 2002; 578: 357.

[17] Titarchuk L, Fiorito R. Spectral index and quasi-periodic oscillation frequency correlation in black hole sources: observational evidence of two phases and phase transition in black holes. Astrophysc J 2004; 612: 988.

[18] Spitzer LS. Physics of fully ionized gases. dover publications, Inc.: Mineola, New York 1962.

[19] Bisnovatyi-Kogan GS, Lovelace RV. Influence of ohmic heating on advection-dominated accretion flows. Astrophysc J 1997; 486: L43.

[20] Quataert E. Particle heating by alfvenic turbulence in hot accretion flows . Astrophysc J 1998; 500: 978. 
[21] Sharma P, Quataert E, Hammett GW, Stone JM. Electron heating in hot accretion flows. Astrophysc J 2007; 667: 714.

[22] Esin AA, Narayan R, Cui W, Grove JE, Zhang SN. Spectral transitions in cygnus X-1 and other black hole X-ray binaries. ApJ 1998; 505: 854.

[23] Yuan F, Taam RE, Xue Y, Cui W. Hot One-temperature accretion flows revisited. Astrophysc J 2006; 636: 46.

[24] Schödel R, Ott T, Genzel R, et al. A star in a 15.2-year orbit around the supermassive black hole at the centre of the Milky Way. Nature 2002; 419: 694 .

[25] Ghez AM, Salim S, Hornstein SD, et al. Stellar orbits around the galactic center black hole. Astrophysc J 2005; 620: 744.

[26] Melia F, Liu S, Coker R. Polarized millimeter and submillimeter emission from sagittarius $\mathrm{A}^{*}$ at the galactic center. Astrophysc $\mathbf{J}$ 2000; 545: L117.

[27] Melia F, Liu S, Coker R. A Magnetic dynamo origin for the submillimeter excess in sagittarius $A^{*}$. Astrophysc J 2001; 553: 146.

[28] Baganoff FK, Bautz MW, Brandt WN, et al. Rapid X-ray flaring from the direction of the supermassive black hole at the Galactic Centre. Nature 2001; 413: 45

[29] Genzel R, Schödel R, Ott T, et al. Near-infrared flares from accreting gas around the supermassive black hole at the galactic centre. Nature 2003; 425: 934.

[30] Gillessen S, Eisenhauer F, Quataert E, et al. Variations in the spectral slope of sagittarius $A^{*}$ during a near-infrared flare. Astrophysc J 2006; 640: 163L.

[31] Bittner J, Liu S, Fryer CL, Petrosian V. Correlation between flux and spectral index during flares in sagittarius $\mathrm{A}^{*}$. Astrophysc $\mathrm{J}$ 2007; 661: 863 .

[32] Wilms J, Nowak MA, Pottschmidt K, Pooley GG, Fritz S. Long term variability of cygnus X-1. IV. spectral evolution 1999-2004. Astron Astrophysc 2006; 447: 245.

[33] Paczynky B, Wiitta PJ. Thick accretion disks and supercritical luminosities. Astron Astrophysc 1980; 88: 23.

[34] Blandford RD, Begelman MC. On the fate of gas accreting at a low rate on to a black hole. MNRAS 1999; 303: L1.

[35] Hawley JF, Balbus SA. The dynamical structure of nonradiative black hole accretion flows. Astrophysc J 2002; 573: 738.

[36] Agol E, Krolik JH. Magnetic stress at the marginally stable orbit: altered disk structure, radiation, and black hole spin evolution. Astrophysc J 2000; 528: 161.

[37] Boldyrev S. Kolmogorov-burgers model for star-forming turbulence. Astrophysc J 2002; 569:841.

[38] Hawley JF, Gammie CF, Balbus SA. Local three-dimensional magnetohydrodynamic simulations of accretion disks. Astrophysc $\mathbf{J}$ 1995; 440: 742.

[39] Pessah ME, Chan CK, Psaltis D. Local Model for angularmomentum transport in accretion disks driven by the magnetorotational instability. PRL 2006; 97: 221103.

[40] Cho J, Vishniac ET. The generation of magnetic fields through driven turbulence. Astrophysc J 2000; 538: 217.

[41] Vestuto JG, Ostriker EC, Stone JM. Spectral properties of compressible magnetohydrodynamic turbulence from numerical simulations. Astrophysc J 2003; 590: 858.

[42] Mukhopadhyay B. Description of pseudo-newtonian potential for the relativistic accretion disks around kerr black holes. Astrophysc J 2002; 581: 427.

[43] Kolmogorov AN. The local structure of turbulence in incompressible viscous fluid for very large reynolds' numbers . Dokl. Akad. Nauk SSSR 1941; 30: 301.

[44] Kraichnan RH. Inertial-range spectrum of hydromagnetic turbulence. Phys. Fluids 1965; 8: 1385.
[45] Zhou Y, Matthaeus WH. Transport and turbulence modeling of solar wind fluctuations. JGR 1990; 95: 14881.

[46] Jiang YW, Liu S, Petrosian V. Cascade and damping of alfvéncyclotron fluctuations: application to solar wind turbulence. Astrophysc J 2009; 698: 163.

[47] Petrosian V, Yan H, Lazarian A. Damping of magnetohydrodynamic turbulence in solar flares. Astrophysc J 2006; 644: 603.

[48] King AR, Pringle JE, Livio M. Accretion disk viscosity: how big is alpha? MNRAS 2007; 376: 1740.

[49] Blandford R, Eichler D. Particle acceleration at astrophysical shocks - a theory of cosmic-ray origin. Phys Report 1987; 154: 1.

[50] Liu S, Petrosian V, Melia F, Fryer CL. A Testable stochastic acceleration model for flares in sagittarius A*. ApJ 2006; 648: 1020.

[51] Petrosian V, Liu S. Stochastic acceleration of electrons and protons. i. acceleration by parallel propagating waves. Astrophysc J 2004; 610: 550.

[52] Wolfe B, Melia F. Covariant kinetic theory with an application to the coma cluster. Astrophysc J 2006; 638: 125.

[53] Liu S, Melia F, Petrosian V, Fatuzzo M. Stochastic acceleration in the galactic center HESS source. Astrophysc J 2006; 647: 1099.

[54] Aitken DK, Greaves J, Chrysostomou A, et al. Detection of polarized millimeter and submillimeter emission from sagittarius A*. Astrophysc J 2000; 534: L173.

[55] Quataert E, Gruzinov A. Constraining the accretion rate onto sagittarius $A^{*}$ using linear polarization. Astrophysc J 2000; 545: 842.

[56] Liu S, Melia F. An accretion-induced x-ray flare in sagittarius A*. Astrophysc J 2002; 566: L77.

[57] Shen ZQ, Lo KY, Liang MC, Ho PTP, Zhao JH. A size of $1 \mathrm{AU}$ for the radio source $\mathrm{Sgr} \mathrm{A}^{*}$ at the centre of the Milky Way. Nature 2005; 438: 62.

[58] Liu S, Qian L, Wu X-B, Fryer CL, Li H. The nature of linearly polarized millimeter and submillimeter emission in sagittarius $\mathrm{A}^{*}$. Astrophysc J 2007; 668: L127.

[59] Rybicki G, Lightman A. Radiative processes in astrophysics. New York: Wiley 1979.

[60] Narayan R, Yi I. Advection-dominated accretion: underfed black holes and neutron stars. Astrophysc J 1995; 452: 710.

[61] Meyer-Hofmeister E, Liu BF, Meyer F. Hysteresis in spectral state transitions - a challenge for theoretical modeling. Astron Astrophysc 2005; 432: 181.

[62] Melia F, Coker R. Stellar gas flows into a dark cluster potential at the galactic center. Astrophysc J 1999; 511: 750.

[63] Petrosian V. Synchrotron emissivity from mildly relativistic particles. Astrophysc J 1981; 251: 727.

[64] Mahadevan R, Narayan R, Yi I. Harmony in electrons: cyclotron and synchrotron emission by thermal electrons in a magnetic field. Astrophysc J 1996; 465: 327.

[65] Readhead ACS. Equipartition brightness temperature and the inverse Compton catastrophe. Astrophysc J 1994; 426: 51.

[66] Barrio FE, Done C, Nayakshin S. On the accretion geometry of Cyg X-1 in the low/hard state. MNRAS 2003; 342: 557.

[67] Titarchuk L. Generalized comptonization models and application to the recent high-energy observations. Astrophysc J 1994; 434: 570.

[68] Heinz, S, Sunyaev, RA. The non-linear dependence of flux on black hole mass and accretion rate in core-dominated jets. MNRAS 2003; 343: L59.

[69] Popham R, Gammie CF. Advection-dominated accretion flows in the kerr metric. ii. steady state global solutions. Astrophysc J 1998; 504: 419 . 